\title{
Puf1p acts in combination with other yeast Puf proteins to control mRNA stability
}

\author{
RANDI J. ULBRICHT and WENDY M. OLIVAS ${ }^{1}$ \\ Department of Biology, University of Missouri-St. Louis, St. Louis, Missouri 63121, USA
}

\begin{abstract}
The eukaryotic Puf proteins bind 3' untranslated region (UTR) sequence elements to regulate the stability and translation of their target transcripts, and such regulatory events are critical for cell growth and development. Several global genome analyses have identified hundreds of potential mRNA targets of the Saccharomyces cerevisiae Puf proteins; however, only three mRNA targets for these proteins have been characterized thus far. After direct testing of nearly 40 candidate mRNAs, we established two of these as true mRNA targets of Puf-mediated decay in yeast, HXK1 and TIF1. In a novel finding, multiple Puf proteins, including Puf1p, regulate both of these mRNAs in combination. TIF1 mRNA decay can be stimulated individually by Puf1p and Puf5p, but the combination of both proteins is required for full regulation. This Puf-mediated decay requires the presence of two UGUA binding sites within the TIF1 3' UTR, with one site regulated by Puf5p and the other by both Puf1p and Puf5p. Alteration of the UGUA site in the tif1 $3^{\prime}$ UTR to more closely resemble the Puf3p binding site broadens the specificity to include regulation by Puf3p. The stability of the endogenously transcribed HXK1 mRNA, cellular levels of Hxk1 protein activity, and HXK1 3' UTR-directed decay are affected by Puf1p and Puf5p as well as Puf4p. Together these results identify the first mRNA targets of Puf1p-mediated decay, describe similar yet distinct combinatorial control of two new target mRNAs by the yeast Puf proteins, and suggest the importance of direct testing to evaluate RNA-regulatory mechanisms.
\end{abstract}

Keywords: Puf; decay; stability; mRNA; yeast; 3' UTR

\section{INTRODUCTION}

The regulation of mRNA stability is a critical component of post-transcriptional control of gene expression (Guhaniyogi and Brewer 2001; Parker and Song 2004). Modulation of mRNA decay rates is also an efficient method to rapidly alter gene expression in response to cellular changes (Shim and Karin 2002; Lidder et al. 2005; Penelova et al. 2005). The control elements that regulate mRNA stability are commonly found within the $3^{\prime}$ untranslated region (UTR), and multiple classes of RNA-binding proteins have been identified that sequence-specifically bind these elements (Derrigo et al. 2000; Grzybowska et al. 2001).

The Puf protein family is a widely conserved class of RNA-binding proteins with multiple members across eukaryotes (Wickens et al. 2002). Puf proteins play important roles in stem cell maintenance (Forbes and Lehmann

Reprint requests to: Wendy $\mathrm{M}$. Olivas, Department of Biology, University of Missouri-St. Louis, One University Boulevard, St. Louis, MO 63121-4499, USA; e-mail: olivasw@umsl.edu; fax: (314) 516-6233.

Article published online ahead of print. Article and publication date are at http://www.rnajournal.org/cgi/doi/10.1261/rna.847408.
1998; Parisi and Lin 1999; Crittenden et al. 2002; Moore et al. 2003), cell development and differentiation (Murata and Wharton 1995; Zhang et al. 1997; Gamberi et al. 2002; Nakahata et al. 2003), and neuronal plasticity (Menon et al. 2004; Ye et al. 2004). Puf protein functional activity is based on their ability to stimulate deadenylation and decay and/or suppress translation of bound mRNAs (Wreden et al. 1997; Olivas and Parker 2000; Wickens et al. 2002). Regulation of mRNA metabolism by metazoan Puf proteins, such as Pumilio in Drosophila and FBF in Caenorhabditis elegans, requires recruitment of additional protein partners to the mRNA (Sonoda and Wharton 1999, 2001; Kraemer et al. 1999). In Saccharomyces cerevisiae, evidence suggests that Puf proteins directly recruit mRNA decay factors to the mRNA (Goldstrohm et al. 2006; F.A. Lopez Leban, S.S. Houshmandi, and W.M. Olivas, unpubl.).

Puf proteins are characterized by an RNA-binding domain composed of eight imperfect repeats of 36 amino acids plus short flanking regions. This Puf repeat domain folds into an extended crescent-shaped structure, where RNA binding occurs on the inner concave surface and interactions with other proteins utilize the outer convex surface (Edwards et al. 2001; Wang et al. 2002). RNAs 
targeted by Pufs contain a consensus UGUR Puf binding motif in the $3^{\prime}$ UTR, with flanking sequences conferring specificity to distinct Puf proteins (Wickens et al. 2002; Jackson et al. 2004; Bernstein et al. 2005).

S. cerevisiae encodes six Puf proteins (Puflp-Puf6p), though only four have verified roles in modulating mRNA stability and/or translation via $3^{\prime}$ UTR binding. Puf3p promotes deadenylation and decay of COX17 mRNA (Olivas and Parker 2000), Puf4p and Puf5p promote deadenylation and decay of $H O$ mRNA (Tadauchi et al. 2001; Goldstrohm et al. 2006; Hook et al. 2007), and Puf6p regulates translation of ASH1 mRNA (Gu et al. 2004). However, several different microarray studies have identified hundreds of potential mRNA targets of Puf proteins in yeast. One study found 168 mRNAs whose steady-state poly $(\mathrm{A})+$ levels were altered between a wild-type (WT) strain versus a quintuple mutant strain deleted of PUF1 through PUF5 (Olivas and Parker 2000). A second study analyzed mRNAs that physically associated with tagged Puf proteins 1-5, identifying between 40 and 220 mRNAs that associated with each Puf, 90 of which associated with more than one Puf (Gerber et al. 2004). This study also found 10-11-nucleotide (nt) consensus 3' UTR sequence motifs containing UGUA in many of the mRNAs associated with Puf3p, Puf4p, and Puf5p but no motifs common to the mRNAs associated with Puflp or Puf2p (Gerber et al. 2004). A third study identified multiple transcripts whose stabilities were altered between a wild-type strain and a puf $4 \Delta$ strain following transcriptional repression (Grigull et al. 2004). Finally, a computational algorithm, MatrixREDUCE, which utilized genomic sequence data and steady-state gene expression data from a set of $\sim 750$ microarrays, predicted the nucleotide binding specificities and target mRNAs, as well as the condition-specific activities for Puf3p and Puf4p (Foat et al. 2005). Whereas there was some overlap between the target transcripts identified by the different microarray and computational screens, there were many transcripts that were only identified by one screening method. This suggests that any single method is not exhaustive in determining a complete set of Puf targets, and/or there are many false positive or indirect targets in these candidate sets.

Gene ontology analysis of the candidate Puf target mRNAs showed biases in the types of mRNAs associated with each yeast Puf: Puflp and Puf2p with mRNAs encoding membrane-associated proteins, Puf3p with cytoplasmic mRNAs encoding mitochondrial proteins, Puf4p with mRNAs encoding nucleolar ribosomal components, and Puf5p with mRNAs encoding other nuclear components (Gerber et al. 2004; Grigull et al. 2004; Foat et al. 2005). The mRNA targets identified by MatrixREDUCE also showed coordinate changes in transcript abundance in response to different environmental conditions (Foat et al. 2005), providing further evidence for coordinate regulation of specific classes of mRNAs by Puf proteins. However, there is little experimental data showing if these candidate targets are really bound and regulated by Pufs at the level of RNA stability. In fact, Puflp has been implicated as a mitochondrial outer-membrane protein that physically interacts with the Arp2/3 complex to recruit it to the mitochondria (Fehrenbacher et al. 2005). In addition, Puf3p has also been shown to interact with components of the Arp $2 / 3$ complex and with a component of the actin cytoskeleton, contributing to mitochondrial motility and biogenesis (Garcia-Rodriguez et al. 2007). Whether these functions involve RNA binding and stability control is unknown.

To better understand the role of Puf proteins in regulating mRNA metabolism, we tested the stability of several candidate Puf target mRNAs in wild-type versus PUF deletion strains, focusing on targets of Puflp and Puf2 $p$ for which there are no verified targets of Pufmediated decay regulation. Though many of the candidate mRNAs did not appear to be direct targets of Pufs, at least under the conditions tested, we established two new targets of Puf regulation: TIF1 and HXK1. The stabilities of these mRNAs are regulated coordinately by Puflp and Puf5p, with Puf4p also involved in HXK1 stability. These are the first examples of mRNAs whose stabilities are regulated by Puflp. With $H O$ being the only mRNA previously known to be regulated by multiple Pufs, specifically Puf4p and Puf5p (Goldstrohm et al. 2006; Hook et al. 2007), these are the first examples of Puf5p taking on different Puf partners to directly regulate different mRNAs and of an mRNA that is regulated by more than two Puf proteins. The regulation of TIF1 involves two different Puf binding sites in its $3^{\prime}$ UTR, and the stimulation of mRNA decay by these Pufs is condition-specific. We also show that Puflp activity involves recognition of UGUA sequences and their surrounding sequences, demonstrating that Puflp indeed utilizes this conserved binding element like other Puf proteins. In addition, slight modification of nucleotides surrounding the UGUA can allow regulation by Puf3p, but this alteration does not eliminate the ability of Puflp and Puf5p to regulate this mRNA. These results emphasize the importance of direct testing of candidate Puf target mRNAs and provide new insights into how multiple Pufs may act on single targets.

\section{RESULTS}

\section{Analysis of candidate mRNA targets of Puf decay regulation}

To investigate new mRNA targets of Puf protein-mediated decay regulation, we analyzed the yeast transcriptome for 3' UTR elements containing at least one UGUA sequence element. The outcome of this analysis was then crossreferenced with the candidate mRNAs identified by the microarray study comparing steady-state mRNA levels 
between wild-type yeast and a quintuple PUF deletion strain (Olivas and Parker 2000) or with candidates identified by the microarray study that analyzed mRNAs physically associated with Pufs 1-5 (Gerber et al. 2004). We focused our efforts on mRNAs associated with Puf 1, 2, or 5 , and on mRNAs that appeared to act coordinately with other targets in a cellular pathway. For example, PMP1, PMP2, PMP3, and AST1 mRNAs were all associated with Puflp and/or Puf2p and encode membrane-associated proteins involved in proton transport. Overall, we tested 20 mRNA candidates in our decay assay, including nine associated with Puf1p and/or Puf2p, six associated with Puf5p, and eight from the PUF deletion microarray (Table 1).

For decay analysis, transcriptional shutoff assays were performed using strains containing the temperature-sensitive RNA polymerase II mutant (rpb1-1), in which transcription is inhibited by shifting the temperature to $37^{\circ} \mathrm{C}$ (Herrick et al. 1990). Since Puf proteins appear to be active only under certain conditions, and cell density appears to be one factor that influences Puf activity (Foat et al. 2005), cultures were grown to an optical density $\left(\mathrm{OD}_{600}\right)$ of 0.4 or 1.0 prior to transcriptional repression. Decay profiles of each candidate mRNA were then compared between a wildtype PUF strain (WT) and strains deleted either individually of PUFs $1-5$ or a quintuple PUF deletion strain ( $\Delta p u f 1-5)$. For most mRNAs, we detected no changes in half-lives in the PUF deletion strains under the conditions tested (Table 1). COX17 mRNA, a known target of Puf3p regulation, was used as a positive control in these experiments. For many of the mRNAs, including an additional 18 transcripts not listed in Table 1, steady-state mRNA analysis was also performed comparing mRNA levels between WT and individual PUF deletion strains at $\mathrm{OD}_{600}$ of 0.4 or 1.0. Only five transcripts displayed any significant differences in steady-state levels - the HXK1 and TIF1 mRNAs, as well as the PMP1, PMP2, and PMP3 mRNAs (data not shown). Surprisingly, exhaustive half-life analysis of PMP mRNAs in the individual PUF deletion strains that had revealed changes in mRNA steady-state levels showed no changes in mRNA decay rates (Table 1). Overall, these results suggest that (1) there were many false positives and/or indirect target mRNAs identified by the microarray screens; (2) these mRNAs are targeted by Pufs only under particular growth conditions not yet tested; (3)

TABLE 1. RNAs tested for Puf-mediated regulation of mRNA stability

\begin{tabular}{|c|c|c|c|c|c|c|c|c|c|c|c|c|c|}
\hline \multirow[b]{3}{*}{ RNA } & \multirow[b]{3}{*}{ Source } & \multirow{3}{*}{$\begin{array}{c}\text { Physically } \\
\text { associated } \\
\text { Puf }^{a}\end{array}$} & \multicolumn{11}{|c|}{$\mathrm{OD}_{600}^{\mathrm{b}}$} \\
\hline & & & \multicolumn{5}{|c|}{0.4} & \multicolumn{6}{|c|}{1.0} \\
\hline & & & puf1s & puf $2 \Delta$ & puf3 $\Delta$ & puf4 $\Delta$ & puf5 $\Delta$ & puf1 $\Delta$ & puf2 $\Delta$ & puf3 $\Delta$ & puf $4 \Delta$ & puf5 $\Delta$ & $\Delta$ puf1-5 \\
\hline AME1 & $\begin{array}{l}\text { Olivas and Parker (2000); } \\
\text { Gerber et al. (2004) }\end{array}$ & Puf5 & - & - & - & & - & & & & & & \\
\hline CBC2 & Motif search & & - & - & - & - & & & & & & & \\
\hline COX15 & Gerber et al. (2004) & Puf3, Puf5 & & & - & & & & & & & & \\
\hline COX17 & $\begin{array}{l}\text { Olivas and Parker (2000); } \\
\text { Gerber et al. (2004) }\end{array}$ & Puf3 & - & & + & & & - & - & + & - & - & \\
\hline $\mathrm{DHH} 1$ & Gerber et al. (2004) & Puf1, Puf2, Puf5 & - & & - & & - & & & & & & \\
\hline GCN4 & Olivas and Parker (2000) & & & & & & & - & - & - & - & - & - \\
\hline GLK1 & Olivas and Parker (2000) & & & & & & & - & - & - & - & - & - \\
\hline$H X K 1$ & Motif search & & + & & - & + & + & + & - & - & + & + & + \\
\hline$H X K 2$ & Olivas and Parker (2000) & & & & & & & - & & & & & - \\
\hline MIG1 & Gerber et al. (2004) & Puf1, Puf2 & & - & & & & & & & & & \\
\hline MSN2 & Motif search & & - & & & & & & & & & - & - \\
\hline MSN4 & Gerber et al. (2004) & Puf2 & - & - & - & - & - & - & - & & - & - & - \\
\hline NOP1 & Gerber et al. (2004) & Puf1, Puf4, Puf5 & - & - & - & - & - & & & & & & \\
\hline NUP100 & Gerber et al. (2004) & Puf2, Puf5 & - & - & - & - & - & & & & & & \\
\hline PET117 & $\begin{array}{l}\text { Olivas and Parker (2000); } \\
\text { Gerber et al. (2004) }\end{array}$ & Puf3, Puf5 & & - & - & & & & & & & & \\
\hline PMP1 & Gerber et al. (2004) & Puf2 & - & - & - & - & - & & & & & & \\
\hline PMP2 & Gerber et al. (2004) & Puf2 & & & - & - & & & & & & & \\
\hline PMP3 & $\begin{array}{l}\text { Olivas and Parker (2000); } \\
\text { Gerber et al. (2004) }\end{array}$ & Puf1, Puf2 & - & - & - & - & - & & & & & & \\
\hline PUF1 & Gerber et al. (2004) & Puf1, Puf2 & & - & & & & & & & & & \\
\hline TIF1 & Olivas and Parker (2000) & & - & - & - & & - & + & - & - & - & + & + \\
\hline TPK1 & Motif search & & & & & & & & & & & & \\
\hline
\end{tabular}

${ }^{a}$ Puf protein(s) shown to physically interact with particular RNAs are indicated (Gerber et al. 2004).

${ }^{b}$ Northern blots were prepared from transcriptional shutoff experiments of WT and PUF deletion yeast and probed for the indicated RNA. No significant effect on stability compared with WT is indicated by "-." A significant difference in stability compared with WT is denoted by "+." 
there is redundant control of these mRNAs by multiple Pufs; or (4) certain mRNAs are physically associated with Pufs for a purpose not related to mRNA stability. The remainder of this work will focus on investigating HXK1 and TIF1 as targets of Puf-mediated decay regulation.

\section{TIF1 is targeted for mRNA decay by Puf1 and Puf5}

The TIF1 mRNA was originally identified in the microarray screen comparing RNA levels between the WT and $\Delta p u f 1--$ 5 strains, with TIF1 showing a 3.5-fold difference (Olivas and Parker 2000). The TIF1 3' UTR contains two UGUA elements, potential sites of Puf interaction (Fig. 1A). Interestingly, in our steady-state analysis of TIF1, altered RNA levels were detected from cells harvested at the higher cell density, $\mathrm{OD}_{600}=1.0$, but not at $\mathrm{OD}_{600}=0.4$ (data not shown). To investigate the role of Puf proteins in the decay of TIF1, we performed transcriptional shutoff assays at $\mathrm{OD}_{600}=1.0$ to determine its half-life in WT versus PUF deletion strains. We found endogenous TIF1 mRNA to be very stable with a half-life of $>30 \mathrm{~min}$. Our attempts to assay the affect of PUF deletions on half-life were inconsistent, presumably due to the extended duration of stressful conditions in high cell densities required to assay changes in long half-lives. Therefore, to avoid underestimating or missing changes in its decay rate in PUF deletion strains and also to focus on Puf protein control of mRNA decay via the potential 3' UTR binding elements in TIF1, we cloned the 3' UTR of TIF1 mRNA behind the coding region of MFA2. Previous studies have shown that fusion of the 3' UTR of COX17 to the MFA2 ORF is sufficient for Puf-regulated decay of this fusion construct (Jackson et al. 2004). The MFA2/TIF1 3' UTR fusion was expressed from a plasmid under the transcriptional control of the GAL UAS (Decker and Parker 1993). Thus, in addition to a temperature shift to disable the temperature-sensitive RNA polymerase II in these strains, transcription was also inhibited by changing the carbon source from galactose to dextrose.

In WT yeast, the MFA2/TIF1 3' UTR fusion mRNA decayed with a half-life of $7.0 \pm 0.6$ min (Fig. 1B). In

\section{A. UAAGAACUAAGGGAGACAAAAACUUCUUAAAAAAGUAUCUUGUGAGGCUAUCUUGAUAACGUAACAUAUAUUUCUAAA stop AAGUUAUAUAUGCUUUUUGUAUUUAAUUUGUUUUUCUUUUUACAUUCCUAUUAUUCUUCAAAGUCCAAAAGACUCUC site \#1 GGCACGAAGGUUGAAAUACCCUAUACUAAUUGUUUGCUUUGUCUUUUUGUAUAUACCCGAACGUAUCUAUCUGAAAUU site $\# 2$ UUUCAAAUUUAUAAAAAAUAAUAUAUAUAAAAGGAAAAAAAAAAAAAUCGAUGCCCUACAAA}

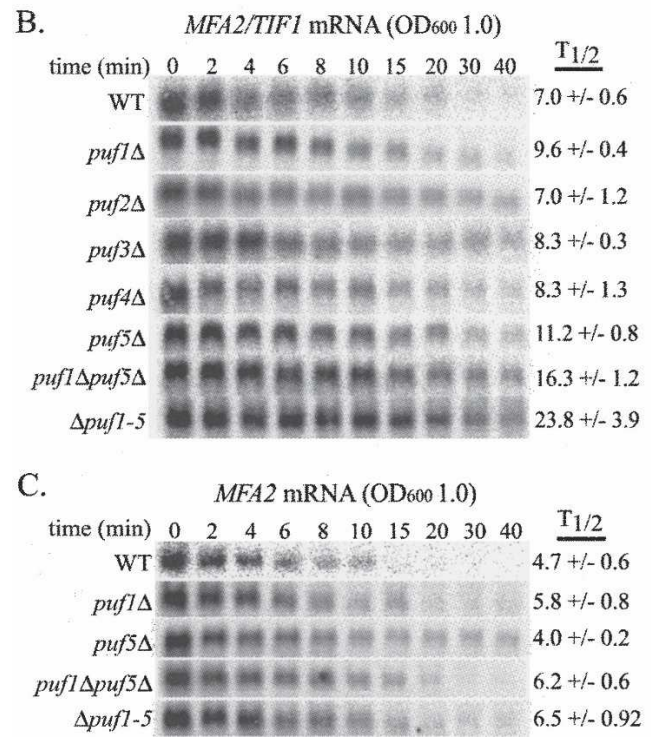

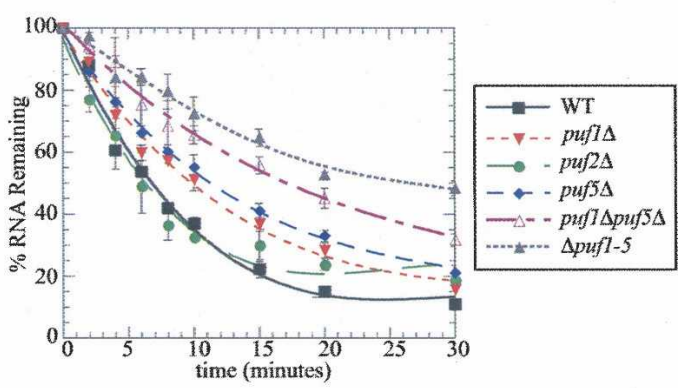

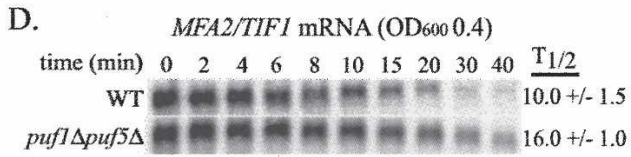

FIGURE 1. TIF1 3' UTR is regulated by Puf1p and Puf5p. (A) Sequence of the TIF1 3' UTR. Underlined regions (sites \#1 and \#2) are proposed sites of Puf interaction. UGUA sequences are in bold. UGUN sequences are shaded gray. The length of the TIF1 3' UTR was estimated through PAGE analysis of the 3' UTR after removal of the poly(A) tail. (B) Decay of MFA2/TIF1 3' UTR fusion mRNA in wild-type (WT), individual PUF deletion, and multiple PUF deletion yeast strains grown to an $\mathrm{OD}_{600}$ of 1.0. Representative Northern blots are presented in the left panel. Data from the Northern analyses are plotted in the right panel. Minutes following transcription repression are indicated above blots and along the $X$ axis of the graph. Decay was measured in the following yeast strains: WT (black, closed square), puf1 $\Delta$ (red, closed upside-down triangle), puf2 $\Delta$ (green, closed circle), puf3 $\Delta$ (not graphed), puf $4 \Delta$ (not graphed), puf5 $\Delta$ (blue, closed diamond), puf1 $\Delta p u f 5 \Delta$ (purple, open triangle), and $\Delta p u f 1-5$ (gray, closed triangle). (C) Decay of MFA2 mRNA with its native $3^{\prime}$ UTR in the same yeast strains and conditions as B. (D) Decay of MFA2/TIF1 mRNA in WT and PUF deletion yeast strains grown to mid-log phase $\left(\mathrm{OD}_{600}\right.$ of 0.4$)$. The estimated $T_{1 / 2}$ is listed to the right of each representative Northern blot. For $B$ and $C$, the error for each data point and/or $T_{1 / 2}$ is the SEM $(n \geq 3)$. For $D$, error is the range $(n=2)$. 
$p u f 2 \Delta$, puf3 $\Delta$, and $p u f 4 \Delta$ yeast strains, the half-life of the MFA2/TIF1 fusion mRNA was similar to that in WT (Fig. 1B). However, compared to WT the MFA2/TIF1 mRNA decayed more slowly in the puf $1 \Delta$ and puf5 $\Delta$ strains, with half-lives of $9.6 \pm 0.4$ and $11.2 \pm 0.8 \mathrm{~min}$, respectively (Fig. 1B). Conversely, MFA2 mRNA with its native $3^{\prime}$ UTR decayed similarly in WT and each PUF deletion strain, including the puf1 $\Delta$ and puf5 $\Delta$ strains (Fig. 1C; data not shown). Thus, both Puflp and Puf5p stimulate mRNA decay via the TIF1 3' UTR.

While the difference in half-lives between WT and either single PUF1 or PUF5 deletion strain was small, there was a more dramatic effect on the MFA2/TIF1 mRNA half-life in the double deletion strain, puf1 $\Delta p u f 5 \Delta$ (Fig. 1B). The halflife in this strain was $16.3 \pm 1.2 \mathrm{~min},>2$-fold slower than WT. Thus, the presence of either Puflp or Puf5p is necessary and sufficient to accelerate mRNA decay through the TIF1 3' UTR, but the presence of both Pufs provides maximal decay stimulation. In contrast, the native MFA2 mRNA decayed similarly between WT yeast and strains deleted of multiple PUF genes (Fig. 1C), again indicating that Pufmediated decay is dependent on elements in the TIF1 3' UTR. The MFA2/TIF1 mRNA decayed even more slowly in the quintuple deletion ( $\Delta p u f 1-5)$ with a half-life of $23.8 \pm$ $3.9 \mathrm{~min}$. Therefore, it is likely that other Pufs may play small compensatory roles in the regulation of TIF1 mRNA decay.

The results shown in Figure 1, B and C, illustrate MFA2/ TIF1 mRNA decay in yeast grown to an $\mathrm{OD}_{600}$ of 1.0 prior to transcription inhibition, as this was the cell density that promoted differences in TIF1 mRNA steady-state levels. When the decay assays were performed under lower cell density (transcription inhibition at an $\mathrm{OD}_{600}$ of 0.4 ), the half-life in the WT strain $(10.0 \pm 1.5 \mathrm{~min})$ was extended compared to the same strain under higher cell density conditions (Fig. 1B,D, cf. WT), indicating that Puf activity is altered under these conditions. The half-life in the puf $1 \Delta$ puf5s strain remained similar between $\mathrm{OD}_{600}=0.4$ $(16.0 \pm 1.0 \mathrm{~min})$ and $\mathrm{OD}_{600}=1.0(16.3 \pm 1.2 \mathrm{~min})$. Decay assays in the individual PUF deletion strains at $\mathrm{OD}_{600}=0.4$ did not show discernible differences in decay of MFA2/TIF1 mRNA (Table 1). These results suggest that Puflp and/or Puf5p activity is condition specific, having greater activity under higher cell density conditions. Previous reports have indicated that Puf proteins are subject to condition-specific regulation. Conditions that are predicted to affect Puf activity include stationary phase and the diauxic shift (Foat et al. 2005). Each of these conditions may account for the altered Puf activity observed in the higher density cultures.

\section{Two UGUA elements in the TIF1 3' UTR are required for Puf1p- and Puf5p-mediated decay}

Previous coprecipitation data indicated that $32 \%$ of the mRNA targets bound to Puf5p contained the consensus sequence of $(\mathrm{U} / \mathrm{A}) \mathrm{UGUA}(\mathrm{A} / \mathrm{U})(\mathrm{C} / \mathrm{U})(\mathrm{A} / \mathrm{U})(\mathrm{U} / \mathrm{A} / \mathrm{G}) \mathrm{UA}$
(Gerber et al. 2004). The first UGUA element in the TIF1 3' UTR, site \#1, diverges only slightly from this consensus sequence, having AU instead of UA at the $3^{\prime}$-most positions (Fig. 1A). The other UGUA element in the TIF1 3' UTR, site \#2, also diverges from the consensus Puf5p-binding sequence at just three positions (Fig. 1A). These two UGUA elements were therefore likely candidates for Puf5p-binding sites in the TIF1 3' UTR. Since no consensus binding sequence had been established for Puflp, we could only postulate based on its similarity to other Puf proteins that it may also have affinity for these UGUA-containing regions of the TIF1 mRNA 3' UTR. If one or both of these sites are required for Puflp/Puf5p-mediated decay, then mutations to these sites should affect the ability of Pufs to stimulate decay of the mutant mRNA. The UGUA of site \#1 was

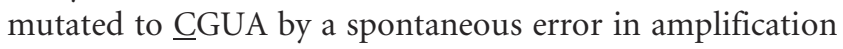
(Fig. 2A). We used PCR-based site-directed mutagenesis to mutate the UGUA of site \#2 in the MFA2/TIF1 3' UTR expression plasmid to ACAC (Fig. 2B). Each of these mutations has previously been shown to eliminate Puf3p binding to its target (Jackson et al. 2004).

The effect of each of these mutations on mRNA stability was measured in the WT and PUF deletion strains. The site \#1 mutant mRNA, MFA2/tif1-1, decayed with a half-life of $8.1 \pm 0.5 \mathrm{~min}$ in WT yeast (Fig. 2A). This half-life is only slightly greater than that of the WT MFA2/TIF1 mRNA $(7.0 \pm 0.6 \mathrm{~min})$, suggesting that disruption of site \#1 is not sufficient to significantly inhibit the ability of Puflp and/or Puf5p to stimulate rapid decay of this transcript. To dissect the role of Puflp and Puf5p in decay, the half-life of the MFA2/tif1-1 mutant mRNA was measured in the PUF deletion strains. MFA2/tif1-1 decayed with a similar half-life in the puf1 $\Delta$ strain $(8.8 \pm 1.0 \mathrm{~min}$ ) as in the WT strain (Fig. $2 \mathrm{~A}$ ), indicating that Puflp-dependent decay requires site \#1. However, the MFA2/tif1-1 mRNA decayed 2.1-fold slower in the puf5 $\Delta$ strain, with a half-life of $17.0 \pm 0.8 \mathrm{~min}$ (Fig. 2A). The decay of MFA2/tif1-1 mRNA was similar in the puf $1 \Delta$ puf5 $\Delta$ double mutant as in the puf5 single mutant (Fig. 2A). Therefore, only Puf5p is required to mediate rapid mRNA decay in the absence of site \#1. Because decay of the MFA2/tif1-1 transcript in the puf5s (Fig. 2A) is similar to decay of WT MFA2/TIF1 in the puf1 $\Delta$ puf5 $\Delta$ strain (Fig. 2B), site \#1 appears essential for the ability of Puflp to stimulate decay of MFA2/TIF1 mRNA, but Puf5p can still stimulate decay via another binding site within the TIF1 3' UTR.

Analysis of the site \#2 mutant mRNA, MFA2/tif1-2, displayed a different decay phenotype. The half-life of this mutant mRNA in the WT strain $(9.9 \pm 0.7 \mathrm{~min})$ was longer than the WT mRNA in the WT yeast strain $(7.0 \pm 0.6 \mathrm{~min})$ but similar to the WT mRNA in either the pufl $\Delta$ (9.6 \pm 0.4 min) or puf5 $\Delta(11.2 \pm 0.8 \mathrm{~min})$ strains (cf. Figs. $2 \mathrm{~B}$ and 1B). This result suggests that whereas site \#2 contributes to decay regulation, Puf1p and/or Puf5p can still partially stimulate decay through another site, likely site \#1. Moreover, decay regulation through site \#2 must be mediated by 

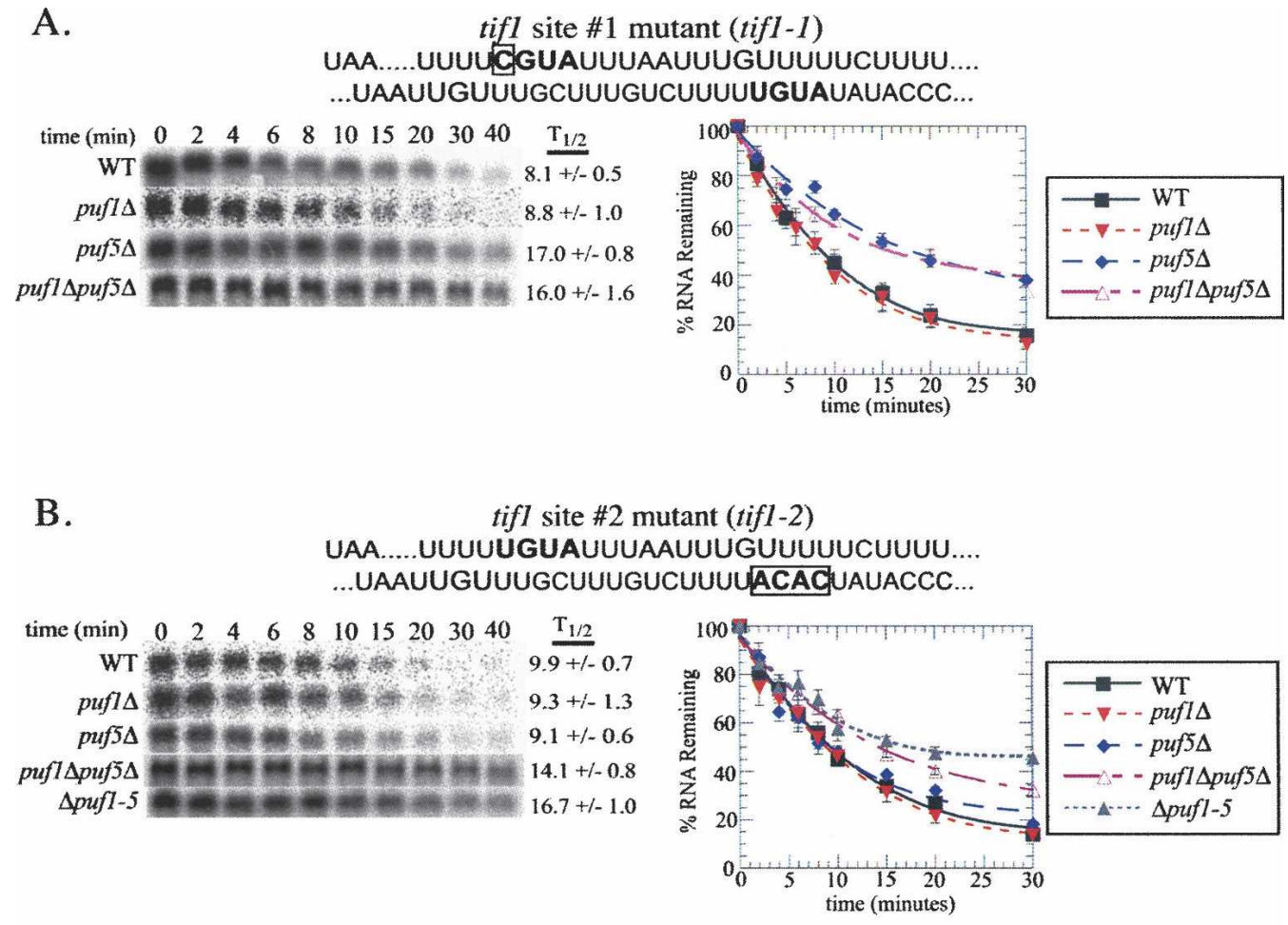

C.

tifl double mutant (tifI-2X)

UAA....UUUUGGUAUUUAAUUUGUUUUUCUUUU... ... UAAUUGUUUGCUUUGUCUUUUACACUAUACCC...
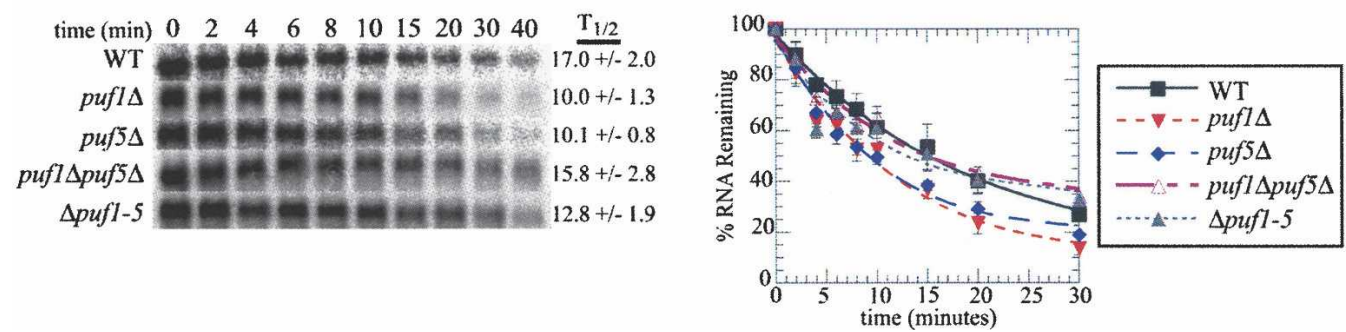

FIGURE 2. Two UGUA sites are required for Puflp- and Puf5p-regulated decay of MFA2/TIF1 mRNA. (A) Decay of MFA2/tif1-1 3' UTR fusion mRNA, where site \#1 is mutated (box). (B) Decay of MFA2/tif1-2 3' UTR fusion mRNA, where site \#2 wis mutated (box). (C) Decay of MFA2/ tif1-2x 3' UTR fusion mRNA where both sites \#1 and \#2 are mutated (box). Representative Northern blots for each mRNA in each strain are presented in the left panels. The estimated $T_{1 / 2}$ is listed to the right of each Northern blot. Data from the Northern analyses are plotted in the right panels. Minutes following transcription repression are indicated above each set of blots and along the $X$-axis of the graphs. Error for each time point and $T_{1 / 2}$ is the SEM $(n \geq 3)$. Decay was measured in the same strains as in Figure 1.

Puf5p, since Puflp-dependent decay depends solely on site \#1. To determine whether it is only Puflp or both Puflp and Puf5p that stimulate decay through site \#1, the mRNA half-life of MFA2/tif1-2 was analyzed in each single deletion strain, the double puf1 $\Delta p u f 5 \Delta$ strain, and the $\Delta p u f 1-5$ strain. While deletion of either PUF1 or PUF5 had no further stabilizing effect on the mRNA, with half-lives of $9.3 \pm 1.3$ and $9.1 \pm 0.6 \mathrm{~min}$, respectively, the half-life in the double puf $1 \Delta$ puf $5 \Delta$ mutant strain was slowed to $14.1 \pm 0.8$ min. Moreover, the half-life in the $\Delta p u f 1-5$ strain (16.7 \pm $1.0 \mathrm{~min})$ was similar to the puf1 $\Delta$ puf $5 \Delta$ mutant half-life (Fig. 2B). These results indicate that Puflp and Puf5p are each capable of regulating mRNA decay via the TIF1 site \#1, and other Puf proteins have little affect on this decay.

If no other sites are involved in Puflp and Puf5p regulation of the TIF1 $3^{\prime}$ UTR, the combination of site $\# 1$ and \#2 mutations should eliminate decay regulation. As expected, decay of this double site mutant mRNA, MFA2/ tif1-2x, in WT yeast appeared unregulated by Pufs, with a half-life of $17.0 \pm 2.0 \mathrm{~min}$ (Fig. 2C). This decay is similar to the half-lives of both WT MFA2/TIF1 mRNA in the puf $1 \Delta$ puf $5 \Delta$ strain (Fig. 1B, $16.3 \pm 1.2 \mathrm{~min}$ ) and of MFA2/ tif1- $2 x$ in the puf1 $\Delta$ puf $5 \Delta$ strain (Fig. 2 C, $15.8 \pm 2.8 \mathrm{~min}$ ). Together, these data provide evidence that these two 
UGUA sites are the primary targets for Puflp/Puf5pmediated decay stimulation. Unexpectedly, decay of the MFA2/tif1-2x mutant mRNA was accelerated in the singledeletion puf $1 \Delta$ and puf5 $\Delta$ strains, with half-lives of $10.0 \pm$ 1.3 and $10.1 \pm 0.8 \mathrm{~min}$, respectively (Fig. $2 \mathrm{C}$ ). One possible explanation for these results is that various Puf proteins may be able to bind the $3^{\prime}$ UTR at alternate locations when the two UGUA sites are mutated. Alternatively, Puf proteins may normally bind these alternate sites, but only upon mutation of the UGUA sites does this binding have a functional effect on the mRNA. Studies with the C. elegans protein FBF-1 have found that this Puf can bind to different UGUN sequences, where $\mathrm{N}$ is $\mathrm{A}$, $\mathrm{U}$, or $\mathrm{G}$ (Bernstein et al. 2005). The TIF1 3' UTR contains two UGUU sites, a UGUC, and a UGUG (Fig. 1A, shaded gray). Indeed, Puf proteins can be somewhat promiscuous in their binding, with multiple Puf proteins able to bind the same site, albeit with different affinities, but only specific Pufs are able to promote an in vivo decay effect (Houshmandi and Olivas 2005; data not shown). In the case of the TIF1 3' UTR, mutation of sites \#1 and \#2 may have altered the structure or sequence contexts of these alternate sites for better access by Pufs or other regulatory factors. If either Puf1p or Puf5p is absent, this may tilt the balance of other proteins gaining access to these alternate sites, thereby impacting the stability of the mRNA. In fact, decay of the MFA2/tif1-2x mRNA in the $\Delta p u f 1-5$ strain (half-life of $12.8 \pm 1.9 \mathrm{~min}$ ) is faster than the WT mRNA in the $\Delta p u f 1-5$ strain (half-life of $23.8 \pm 3.9 \mathrm{~min}$ ), supporting a hypothesis that mutation of sites \#1 and \#2 has altered the intrinsic stability of the mRNA in the absence of Pufs.

\section{Specificity of TIF1 mRNA can be altered to include regulation by Puf3p}

Upon comparison of the TIF1 UGUA sites important for Puflp and Puf5p regulation to the UGUA sites important for COX17 regulation (Jackson et al. 2004), we found that the experimentally verified 12-nt COX17 mRNA Puf3p element (site \#1) differs from TIF1 3' UTR site \#1 by only 4 nt (Fig. 3A; Jackson et al. 2004). To determine if these $4 \mathrm{nt}$ determine the specificity of Puf3p for its target mRNAs, we first altered the TIF1 site \#1 UGUA site in the MFA2/tif1-2 construct to resemble the COX17 Puf3 element. If these 4 nt are responsible for recruiting Puf3p, we expect that the stability of the new construct, named MFA2/tif1-P3E, will be regulated by Puf3p. In fact, the half-life of MFA2/tif1P3E mRNA is extended twofold to $15.7 \pm 1.8 \mathrm{~min}$ in puf $3 \Delta$ yeast compared to WT yeast (Fig. 3B, $7.2 \pm 1.3 \mathrm{~min}$ ). Thus, by altering only $4 \mathrm{nt}$ surrounding the TIF1 3' UTR UGUA, we have enabled regulation by Puf3p. Interestingly, the MFA2/tif1-P3E mRNA half-life in the puf1 $\Delta p u f 5 \Delta$ strain is $13.0 \pm 1.1 \mathrm{~min}$, similar to that of the puf $3 \Delta$ yeast (Fig. 3B), suggesting that Puflp and/or Puf5p maintain their ability to regulate this mRNA despite the changes to the binding site.

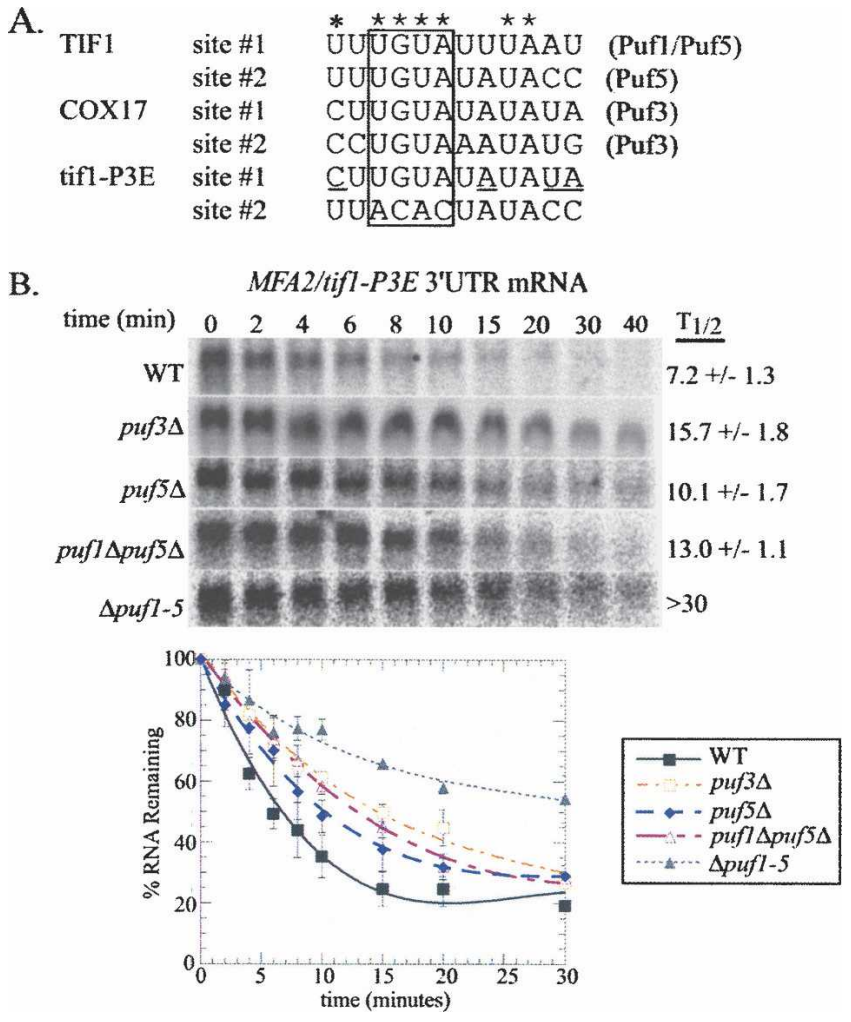

FIGURE 3. TIF1 3' UTR can be modified for regulation by Puf3p. (A) Shown are TIF1 and COX17 3' UTR 12-nt Puf elements, including and surrounding the core UGUA (box). The Puf responsible for regulating each site is listed to the right of each site. A star above the nucleotide position denotes that this position is identical in all four Puf sites. The asterisk indicates the nucleotide position that is identical in both Puf3 sites but differs in both Puf1 and Puf5 sites. The four positions mutated from tif1-2 3' UTR to produce tif1-P3E 3' UTR are underlined. (B) Decay of MFA2/tif1-P3E mRNA in WT and PUF deletion strains. Representative Northern blots from each strain are presented in the top panel. The estimated $T_{1 / 2}$ is listed to the right of each Northern blot. In the bottom panel, the average of the data from the Northern blots is plotted. Minutes following transcription repression are indicated above each set of blots and along the $X$-axis of the graphs. Error for each data point and $T_{1 / 2}$ is the SEM $(n \geq 3)$. Symbols for each strain are the same as in Figure 1, except puf $3 \Delta$ (orange, open square).

In fact, the MFA2/tif1-P3E mRNA half-life in yeast lacking all five PUFs is $>30 \mathrm{~min}$ (Fig. 3B), further suggesting that Pufs other than Puf3p stimulate decay of MFA2/tif1-P3E mRNA. Decay of the mRNA in the puf5s strain is $10.1 \pm$ $1.7 \mathrm{~min}$, intermediate to WT and puf $1 \Delta$ puf $5 \Delta$ yeast (Fig. $3 \mathrm{~B})$, suggesting that Puflp and Puf5p both contribute to the decay of MFA2/tif1-P3E. Therefore, binding site recognition by Puflp and Puf5p appears to be fairly flexible.

\section{HXK1 mRNA decay is regulated by Puf1p, Puf5p, and Puf4p}

To analyze other potential targets of Puf-regulated decay, the same Northern blots of mRNAs from transcriptional 
shutoff experiments that illustrated decay of MFA2 and MFA2/TIF1 mRNAs (Fig. 1, RNA harvested at $\mathrm{OD}_{600}$ of 1.0) were probed for the endogenously transcribed GLK1, HXK2, MSN2, and MSN4 mRNAs. Like TIF1 and COX17, the GLK1 and HXK2 mRNAs were identified as differentially expressed in the original PUF deletion microarray, MSN4 was found physically associated with Puf2p, and MSN2 was a functionally related gene (Table 1). All of these mRNAs contain potential Puf-binding elements in their $3^{\prime}$ UTRs. However, the half-lives of these transcripts were not significantly affected by PUF deletions (Table 1). We also tested the decay of $H X K 1$ mRNA. While this mRNA was not identified in any of the microarray experiments, Hxk1p function, regulation, and expression are related to the GLK1, HXK2, MSN2, and MSN4 genes. The hexokinases Hxk1p and Hxk2p are involved in regulating transcription of the GLK1, HXK2, and HXK1 genes in response to glucose (Rodriguez et al. 2001). The transcription factors Msn2p and Msn4p activate transcription of the GLK1 and $H X K 1$ genes in response to stress (Boy-Marcotte et al. 1998). The HXK1 3' UTR contains multiple conserved Puf- binding elements, further suggesting it may be a target of Puf-mediated decay.

Decay of $H X K 1$ mRNA was markedly slower in the $p u f 1 \Delta, p u f 4 \Delta$, and $p u f 5 \Delta$ strains relative to WT, $p u f 2 \Delta$, and puf3 $\Delta$ strains (Fig. 4A). The decay pattern of HXK1 mRNA is irregular, increasing in abundance after a temperature shift for $4 \mathrm{~min}$ in WT but after $\sim 10 \mathrm{~min}$ in puf1 $\Delta$, puf $4 \Delta$, and $p u f 5 \Delta$ strains, before finally decreasing in abundance (Fig. 4A). Other mRNAs, including TIF1 and MFA2, probed on these same blots showed no delay in decay, indicating a successful inhibition of transcription. A similar pattern has been observed for certain mRNAs that are particularly responsive to cell stress or involved in the heatshock response (Adams and Gross 1991; Taylor et al. 2005; Aragon et al. 2006). From these decay patterns, it appears as though $H X K 1$ mRNA is stabilized in the puf1 $\Delta$, puf $4 \Delta$, and $p u f 5 \Delta$ strains and even more so in the $\Delta p u f 1-5$ strain. It is notable that the decay patterns observed from higher optical density $\left(\mathrm{OD}_{600}=1.0\right)$ (Fig. $\left.4 \mathrm{~A}\right)$ remain consistent at a lower optical density $\left(\mathrm{OD}_{600}=0.4\right)$ (data not shown). These results suggest that Puflp, Puf4p, and Puf5p are
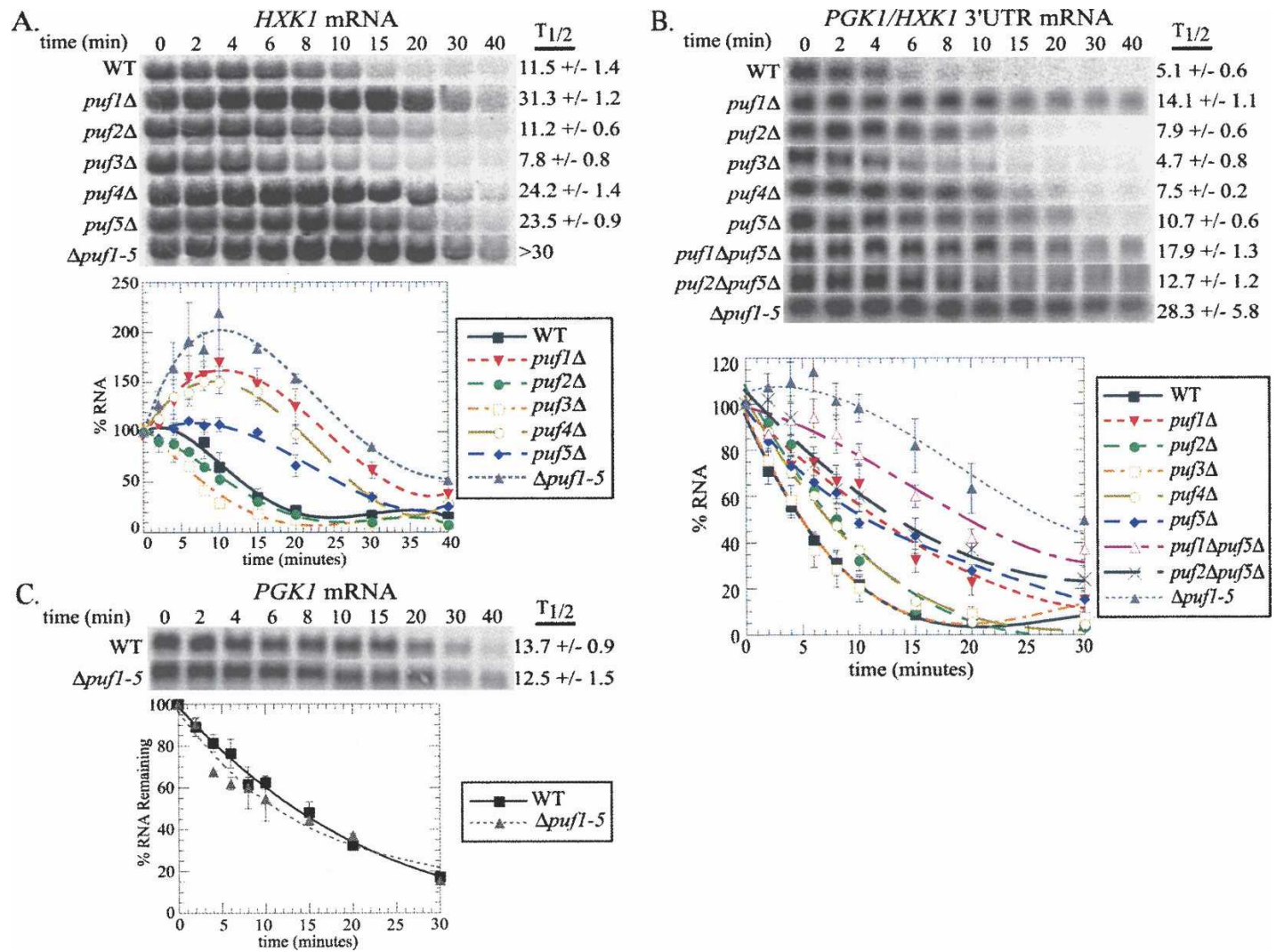

FIGURE 4. HXK1 mRNA is regulated by multiple Puf proteins. (A) Northern blot analyses of endogenously transcribed HXK1 mRNA. Northern blots from Figures 1 and 2 were re-probed for HXK1 mRNA. (B) Decay of PGK1/HXK1 mRNA. (C) Decay of the control PGK1 mRNA. Representative Northern blots are presented in the top panels. The estimated $T_{1 / 2}$ is listed to the right of each Northern blot. Graphical representation of the average of the data from the Northern blots is presented in the lower panels. Minutes following transcription repression are indicated above each set of blots and along the $X$-axis of the graphs. The symbols used here are as in Figures 1-3, except for puf4 $\Delta$ (olive, open circle) and puf $2 \Delta$ puf5 $\Delta$ (black $\times)$. Error for each time point and $T_{1 / 2}$ is the SEM $(n \geq 3)$. 
destabilizing $H X K 1$ full-length mRNA in vivo under both conditions tested.

Due to the irregular decay pattern, the above experiments alone cannot completely eliminate the possibility that Pufs have some effect on $H X K 1$ expression unrelated to mRNA decay. Exclusion of HXK2, GLK1, MSN2, and MSN4 mRNAs as targets of Puf-mediated decay rules out many possible indirect effects Pufs may play via $H X K 1$ regulators; however, there remain other possible factors. To better determine Puf-specific effects on HXK1 mRNA decay, we fused the $3^{\prime}$ UTR of $H X K 1$ to a truncated PGK1 coding region (see Materials and Methods). The expression of this $P G K 1 / H X K 1$ mRNA is regulated by GAL UAS, eliminating any transcriptional variations that may occur at the endogenous $H X K 1$ locus and any translational or stability affects of the $H X K 1$ coding region or 5' UTR. As expected from the endogenous $H X K 1$ mRNA decay results, the PGK1/HXK1 3' UTR fusion mRNA decayed similarly in the WT and puf3 $\Delta$ strains with half-lives of $5.1 \pm 0.6$ and $4.7 \pm 0.8 \mathrm{~min}$, respectively (Fig. 4B). Also expected from our previous results, the $P G K 1 / H X K 1$ mRNA decayed slower in both the puf1 $\Delta$ and puf5 $\Delta$ strains, with half-lives of $14.1 \pm 1.1$ and $10.7 \pm 0.6 \mathrm{~min}$, respectively (Fig. 4B). The PGK1/HXK1 mRNA half-life was also affected by deletion of PUF4 (7.5 $\pm 0.2 \mathrm{~min})$, although not to the same extent as in the puf1 $\Delta$ and puf5 $\Delta$ strains (Fig. 4B). Unexpectedly, the $P G K 1 / H X K 1$ mRNA half-life was also slightly prolonged in the $p u f 2 \Delta$ (7.9 $\pm 0.6 \mathrm{~min})$. The $P G K 1 / H X K 1 \mathrm{mRNA}$ halflife was greatly increased in the $\Delta p u f 1-5$ strain $(28.3 \pm 5.8$ min), whereas the control PGK1 mRNA decayed similarly in both WT and $\Delta p u f 1-5$ strains (Fig. $4 \mathrm{~B}, \mathrm{C}$ ). Thus, like the TIF1 mRNA, decay of HXK1 mRNA is accelerated by both Puflp and Puf5p. However, in a unique fashion, Puf4p and Puf2p also stimulate $H X K 1$ mRNA decay.

Because the half-life of $P G K 1 / H X K 1 \mathrm{mRNA}$ is two- to threefold longer in the $\Delta p u f 1-5$ strain than any individual PUF deletion strain, we can assume that more than one Puf protein is acting on $H X K 1$ mRNA under these conditions. Previous studies have shown that Puf5p acts in combination with Puf4p to regulate HO mRNA (Hook et al. 2007), and our studies have shown that Puf5p acts in combination with Puflp to regulate decay of TIF1 mRNA. To determine whether Puf5p acts in combination with other Puf proteins to regulate $H X K 1$ mRNA decay, we tested decay of PGK1/ HXK1 mRNA in puf1 $\Delta p u f 5 \Delta$ and puf2 $\Delta$ puf5 $\Delta$ yeast. Compared to the single puf5 $\Delta$ strain $(10.7 \pm 0.6 \mathrm{~min})$ and $p u f 1 \Delta$ strain $(14.1 \pm 1.1 \mathrm{~min})$, the half-life was indeed extended in the puf1 $\Delta p u f 5 \Delta$ double deletion strain (17.9 \pm $1.3 \mathrm{~min}$ ) (Fig. 4C). Therefore, similarly to TIF1 mRNA, regulation of $H X K 1$ mRNA by Puf5p is functioning in combination with Puflp. The half-life of PGK1/HXK1 mRNA was not significantly affected in the puf $2 \Delta p u f 5 \Delta$ strain $(12.7 \pm 1.2 \mathrm{~min})$ compared to the puf5 $\Delta$ strain (10.7 $\pm 0.6 \mathrm{~min}$ ) (Fig. 4C). Thus, it appears that Puf2p and Puf5p do not act in combination to stimulate HXK1
mRNA decay. However, it is possible that, due to the small role of Puf2p-mediated decay, an additive change in halflife in the double deletion is difficult to detect. Because the half-life in the puf2 $\Delta p u f 5 \Delta$ strain $(12.7 \pm 1.2 \mathrm{~min})$ and even in the puf1 $\Delta$ puf5 $\Delta$ strain $(17.9 \pm 1.3 \mathrm{~min})$ was significantly less than the $\Delta p u f 1-5$ strain $(28.3 \pm 5.8$ min), we postulate that Puf4p acts in combination with Puf5p and Puflp to regulate HXK1 mRNA.

\section{Hxk1p is up-regulated in PUF deletion yeast}

To test the effects of Puf proteins on $H X K 1$ gene expression at the cellular level, the activity of Hxklp was measured in WT and PUF deletion strains. The yeast hexokinases Glk1p, Hxk1p, and Hxk2p functionally overlap in that they each phosphorylate glucose. Hxk1p and Hxk2p also phosphorylate fructose (Walsh et al. 1991; Gancedo et al. 1977). However, Hxklp prefers fructose to glucose phosphorylation 3:1, whereas Hxk2p phosphorylates fructose and glucose equally (Walsh et al. 1991). Thus, the stabilization of HXK1 mRNA should result in an increase in Hxk1p and fructose phosphorylation. We measured the amount of fructose phosphorylation based on the coupled reactions of fructose phosphorylation by hexokinase (Hxklp) and the reduction of NADP to NADPH by G6PDH (Fig. 5A). The

A.

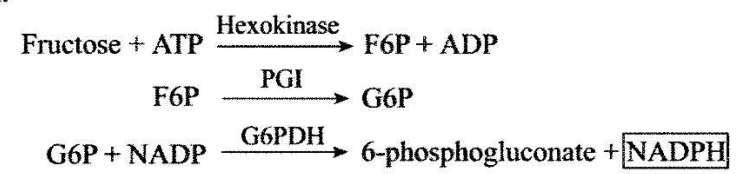

B.

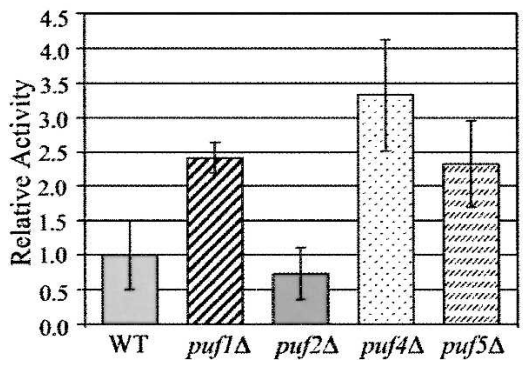

FIGURE 5. Hxklp activity is upregulated in PUF deletion yeast. (A) Outline of NAD-linked assay to measure activity of Hxk1p (hexokinase). Hxklp phosphorylates fructose to make fructose-6-phosphate (F6P). F6P is isomerized to glucose-6-phosphate (G6P) by phosphoglucosisomerase (PGI), and then glucose-6-phosphate dehydrogenase (G6PDH) catalyzes the reduction of NADP into NADPH. Thus, the amount of NADPH produced is dependent on the abundance of Hxk1p in cell extracts. NAPDH production is measured by the change in absorbance at $340 \mathrm{~nm}$. (B) Relative Hxklp enzyme activity in the absence of Puf proteins. Enzymatic activity was determined from puf1 $\Delta$ (diagonal stripes), puf $4 \Delta$ (dotted), puf5 $\Delta$ (hatched), and puf $2 \Delta$ (gray) strains and compared to WT (light gray) yeast. WT and PUF deletion yeast were grown to an $\mathrm{OD}_{600}$ of 1.0 and then harvested, and the lysates were subjected to the described enzyme assay. Enzyme activity $(\mathrm{U} / \mathrm{mL})$ was calculated and is expressed relative to WT. Error bars represent $\mathrm{SD}$. 
production of $\mathrm{NADPH}$ is measured by a change in absorbance at $340 \mathrm{~nm}$. Using these methods, the relative activity of Hxk1p in yeast extracts from WT, puf1 $\Delta$, puf $2 \Delta$, $p u f 4 \Delta$, and puf $5 \Delta$ strains was determined. As seen in Figure 5B, Hxk1p activity was up-regulated 2.4- and 2.3-fold, respectively, in puf $1 \Delta$ and puf5 $\Delta$ strains and 3.3-fold in the puf $4 \Delta$ strain versus wild-type levels. However, there was not a significant difference in activity between the WT and puf $2 \Delta$ strains. These results show that an increased level of protein activity correlates to increased transcript stability in the absence of Puflp, Puf4p, or Puf5p. Hxk1p activity was slightly elevated in the puf $4 \Delta$ strain compared to the puf $1 \Delta$ and $p u f 5 \Delta$ strains; however, this difference is not significant.

It is interesting that, despite destabilization of PGK1/ HXK1 mRNA by Puf2p, neither the HXK1 full-length mRNA decay nor the Hxklp activity appears to be affected by Puf2p. Moreover, the slight stabilization of PGK1/HXK1 mRNA in the absence of PUF4 does not coordinate with the more drastic effects of the puf $4 \Delta$ seen in tests of the fulllength HXK1 mRNA and Hxk1p activity. We suspect that these apparent discrepancies can be explained by unknown affects of the HXK1 promoter, coding region, and/or 5' UTR. Thus, it is possible that, in addition to mRNA decay, Pufs play direct or indirect roles in transcription, translation, and/or cellular availability of the HXK1 transcript.

\section{Puf repeat domains (RDs) bind to the HXK1 3' UTR in vitro}

To determine whether Puf proteins bind to the HXK1 mRNA 3' UTR, in vitro binding assays were performed with in vitro-transcribed and radiolabeled HXK1 mRNA 3' UTR incubated with Puf repeat domains (RDs) tagged with glutathione $S$-transferase (GST) purified from Escherichia coli (Fig. 6A). The RDs of multiple Puf proteins, including yeast Puf3RDp and Puf5RDp, are sufficient for both in vitro binding and in vivo regulation of their targets (Jackson et al. 2004, Houshmandi and Olivas 2005). Following incubation, RNA-protein reactions were UV cross-linked and treated with RNase, resulting in the RNA label attached to the Puf protein if bound to the RNA. Figure 6B demonstrates that GST-tagged Puf1RDp, Puf2RDp, Puf3RDp, and Puf5RDp bind to full-length HXK1 3' UTR. None of these proteins, except for Puf3RDp, were able to bind the COX17 3' UTR (the known target of Puf3p), demonstrating the specificity of binding to the HXK1 3' UTR (data not shown). Puf4RDp was not tested because we were unable to purify stable protein from $E$. coli. The HXK1 3' UTR contains three UGUA elements as candidate Puf-binding sites. Restriction digest of the HXK1 template with Ssp1 truncates the 3' UTR to contain only one UGUA Puf-binding element (Fig. 6A). This truncated RNA was still able to interact with GST-tagged Puf2RDp, Puf3RDp, and Puf5RD but not Puf1RDp (Fig. 6B). These results verify that the HXK1 $3^{\prime}$ UTR is capable of binding
A.

$\frac{\text { UAAGAAAAAAAUGUAAUGAAAUAUAAUGUGUUUUUCCCCUC }}{\text { stop }}$
cCUUAAUAUUAUUUUCUUAUGAGUGAUGCCCUUAUGUUUU
UUUUGCGGUCUAGUAUAUGUAAAUAUAGACACACACAUAUA
Site \#2
UAUAUAUUUAUGUGUAUAAACCUAGCUAAAUAACAUUUU
Site \#3
UAGAUUGUUAUUAUAUAUUUUUCC

B.

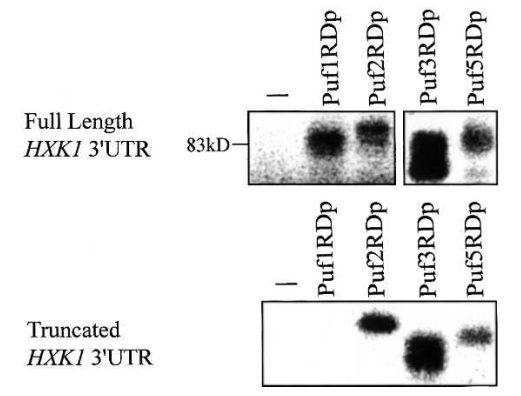

FIGURE 6. Puf repeat domains bind to HXK1 $3^{\prime}$ UTR in vitro. (A) Sequence of the HXK1 $3^{\prime}$ UTR. UGUA-containing regions are underlined and labeled as site \#1, site \#2, and site \#3. (B) Truncated HXK1 3' UTR (lower panel) was transcribed from template cut with SspI, whose location is indicated by an arrow. The length of the $3^{\prime}$ UTR was estimated through PAGE analysis of HXK1 $3^{\prime}$ UTR after removal of the poly $(\mathrm{A})$ tail. In vitro binding assays of radiolabeled transcripts in the presence or absence (-) of GST-PufRDp were UV cross-linked and treated with RNase. Radiolabeled proteins shown in the SDS-polyacrylamide gel represent an interaction between the GST-PufRDp and the transcript.

Puf proteins. Moreover, Puf1RDp likely requires one or both of the latter two UGUA sites in the 3' UTR for activity. These data also reinforce the idea that Puf protein binding is fairly promiscuous, with Puf proteins such as Puf3RDp able to bind this target in vitro, without comparable function in vivo. Similar results were seen with in vitro binding to the TIF1 3' UTR, where Puf1RDp, Puf2RDp, Puf3RDp, and Puf5RDp were all able to bind (data not shown).

\section{DISCUSSION}

Several global microarray studies have identified hundreds of candidate mRNA targets of the yeast Puf proteins. In this work, a closer examination of a subset of candidates has established two mRNAs, TIF1 and HXK1, as direct targets of Puf-mediated decay regulation. For each of these mRNAs, multiple Puf proteins are involved in regulation. For TIF1 mRNA, Puflp and Puf5p are both required for full decay stimulation. For HXK1 mRNA, Puflp, Puf4p, and Puf5p all play a part in decay stimulation and ultimately regulate Hxk1p function. For both of these mRNA targets, the absence of one Puf regulator is sufficient for a partial decay phenotype. The $H O$ mRNA is the only other documented example of a transcript that is regulated by more than one yeast Puf protein, with both Puf4p and 
Puf5p required for maximal stimulation of deadenylation (Goldstrohm et al. 2006; Hook et al. 2007). With just these three examples, it is intriguing that Puf5p is the common Puf acting together in some combination with Puflp and/or Puf4p. It is also clear from the decay phenotypes of individual PUF deletions in our studies and previous studies (Hook et al. 2007) that these Pufs are not simply acting redundantly, but also coordinately, to regulate their targets. Since these mRNAs are the only verified targets of Puf1p/Puf5p or Puf1p/Puf4p/Puf5p, and all show combinatorial control by at least two Pufs, such a mechanism is likely a common theme in mRNA decay regulation by the yeast Pufs. Moreover, combinatorial control may be a conserved mechanism of action in higher eukaryotes as well. In C. elegans, the Puf proteins FBF-1 and FBF-2 act redundantly to control the sperm/oocyte switch via regulation of GLD-1 mRNA (Crittenden et al. 2002), while FBF1 and PUF-8 act redundantly to control a different step of this pathway (Bachorik and Kimble 2005).

We hypothesize that for the TIF1 3' UTR, each UGUA site can recruit its respective Puf protein (Puflp or Puf5p for site \#1, or Puf5p for site \#2), which can individually stimulate decay. However, occupation of both sites promotes an even greater rate of decay. This mechanism is similar to both yeast Puf3p binding to two sites in the COX17 mRNA (Jackson et al. 2004) and Drosophila Pumilio binding two sites in the hunchback mRNA (Wharton and Struhl 1991; Curtis et al. 1997). In each case, occupation of one site promotes partial decay stimulation, while activity at both sites is required for maximum decay control.

Since Puf5p can bind both sites in the TIF1 3' UTR, it is curious why Puflp also is needed for decay control. A simple explanation is that the ability of two different Pufs to stimulate decay may ensure that there is sufficient protein in vivo to occupy both sites. Alternatively, since the activity of Puf proteins is dependent on growth conditions (Foat et al. 2005), the ability of two Pufs to act on TIF1 allows for decay regulation under different conditions that might uniquely inactivate one Puf or the other and/or allow the tweaking of the rate of decay under different conditions. In fact, we have already shown that Puf-mediated decay of TIF1 is primarily detected under high versus low cell density. This result seems logical, as TIF1 encodes the translation initiation factor eIF4A, and at low cell density the cells are actively growing and would require high levels of such translation factors. In contrast, as cell growth begins to slow at higher cell density, translation would also be slowed, thus creating a need for decreased stability of the TIF1 transcript. Interestingly, Puf protein control of translation factors may be a common theme, as Drosophila Pumilio has been shown to bind and down-regulate the translation factor eIF4E at the neuromuscular junction (Menon et al. 2004).

That HXK1 mRNA appears to be regulated by at least three Puf proteins (Puf1p, Puf4p, and Puf5p) at any one time, and the fact that HXK1 contains three UGUA sites suggests a simple model in which one Puf binds to each site at any one time. Our RNA-protein cross-linking studies show that Puflp cannot bind truncated HXK1 3' UTR, suggesting that the Puflp binds sites other than site \#1. The relative levels of stabilization may suggest that Puflp and Puf5p bind with greater affinity than Puf2p and Puf4p or that their relative activity under these conditions vary. It is notable that there was a substantial increase in $H X K 1$ mRNA abundance after a temperature shift, even though other transcripts on the same Northern blots showed successful transcriptional repression. Furthermore, deletion of PUF1, PUF4, or PUF5 dramatically increased both the magnitude and duration of this phenotype, with the quadruple PUF deletion having the largest effect. A microarray study in stationary phase $S$. cerevisiae showed that more than 800 mRNAs, many of them involved in stress response, increased in abundance after induction of oxidative stress. This increase in abundance was not due to new transcription but to accumulation of extraction-resistant species of mRNAs prior to initiation of additional stressors (Aragon et al. 2006). HXK1 mRNA was identified in this study, suggesting that its increase in abundance in our study may be due to accumulation in an extractionresistant storage form (Aragon et al. 2006). Since the increase we observe in HXK1 mRNA abundance is dependent on Puf proteins, then in this scenario, Pufs may play a role in storage and/or localization of HXK1 mRNA. Alternatively, the $r p b 1-1$ allele has been observed to allow transcription, to some extent, of heat-shock genes as well as some stress responsive genes (Adams and Gross 1991). Thus, since $H X K 1$ is a stress responsive gene, and the reporter transcript with the HXK1 3' UTR under the control of an alternative promoter largely lacks this phenotype, it is likely that transcription is not fully repressed from the endogenous $H X K 1$ promoter. In this scenario, Pufs may indirectly affect $H X K 1$ transcription. In either case, it is clear that Pufs indeed affect decay of $H X K 1$ mRNA.

It is unclear why Puflp/Puf5p regulation of TIF1 mRNA is dependent on cell density while their regulation of $H X K 1$ in conjunction with Puf4p is not. One hypothesis is that there is a specific stabilizer of TIF1 mRNA in actively growing cells at low density, and this stabilizer overpowers any effects of the Puf proteins. At higher cell density when translation needs to be down-regulated, this stabilizer may become inactive, allowing the Puf proteins to stimulate decay. Alternatively, condition-specific Puf protein activity may be different on distinct mRNA targets due to disparate protein interactions on different $3^{\prime}$ UTRs and/or conditionally regulated activities of other proteins involved in Puf-mediated decay. In addition, a factor may allow for specificity of Puf binding and/or activity in vivo. In line with this idea, we have observed that Pufs bind promiscuously in vitro where such a specificity factor is lacking. 
The TIF1 and HXK1 mRNAs are the first established targets of Puflp. Like other studied Pufs, Puflp acts to stimulate decay of these mRNA targets. In addition, we show for TIF1 that Puflp decay regulation requires the recognition of UGUA elements in the $3^{\prime}$ UTR, supporting a conserved role of this element for Puf binding. While the global analysis of mRNAs associated with Pufs was unable to detect a consensus binding motif in Puflp-associated mRNAs (Gerber et al. 2004), it is possible that the sequences surrounding the UGUA site are not as well conserved or that there were many false positives in the screen that skewed the analysis. The TIF1 site \#1 that was regulated by Puflp does not match any of the known 1011-nt Puf3p, Puf4p, or Puf5p consensus motifs, though it is only 1-3 nt different from any one of those motifs. In fact, while both sites \#1 and \#2 are regulated by Puf5p, each site is 2-3 nt different from the consensus Puf5p binding motif. As a demonstration of the flexibility of the Puf recognition elements, we show that while altering TIF1 mRNA site \#1 to sequences identical to the 12-nt Puf3p-binding motif from COX17 mRNA allows the regulation by Puf3p, these changes do not eliminate the ability of Puf1p and Puf5p to regulate the mRNA. Work with the C. elegans FBF-1 protein predicted that Pufs require at least $22 \mathrm{nt}$ of sequence surrounding the core UGU, and the base identity at each of these positions can contribute to binding specificity (Bernstein et al. 2005). Thus, RNA recognition by Pufs likely entails an optimal sequence context that can tolerate certain combinations of base changes.

The mechanism by which Pufs stimulates decay of an mRNA target is modeled to involve recruitment of mRNA deadenylation and decapping factors by physically binding Pop2p and/or Ccr4p, which interact with other members of the decay complexes (Goldstrohm et al. 2006; Hook et al. 2007; F.A. Lopez Leban, S.S. Houshmandi, and W.M. Olivas, unpubl.). Thus, in our first model for mRNAs regulated by multiple Puf proteins, each additional Puf protein bound to a $3^{\prime}$ UTR would increase the probability of decay machinery recruitment, thereby enhancing the decay rate. In an alternative model, each Puf may preferentially recruit a different set of the decay machinery components, thereby increasing the probability of having all necessary decay components at the mRNA. Neither of these models preclude other possible roles that Pufs may play in altering 3' UTR mRNP structure to make the mRNA more accessible for decay. Future work will determine if Puflp also interacts with components of the mRNA decay machinery and if so, which components are necessary for Puflp-mediated decay.

Our analysis of nearly 40 candidate targets of Puf protein regulation resulted in only two verified mRNAs that are under Puf-mediated decay control. It seems unlikely that we can account for this small percentage simply by categorizing all the remaining candidates as false positives or indirect targets identified in the microarray screens.
Instead, many of the candidate mRNAs may indeed be direct targets of Puf-mediated decay, but the regulation of decay only occurs under particular growth conditions due to either differential activity of the Pufs or differential activity of other regulatory factors. The conditions under which Puf3p and Puf4p are active to regulate mRNA stability were computationally predicted based on steadystate microarray data of candidate target mRNAs (Foat et al. 2005). However, it is not known how growth conditions might affect the activity of the other Pufs. It is also possible that candidate targets are bound by Pufs for processes other than mRNA decay. For example, the PMP mRNAs were not only bound by particular Pufs (Gerber et al. 2004), but we showed they had changes in steady-state levels in some PUF deletions. However, we could not detect any changes in their half-lives under these conditions, suggesting that Pufs may be acting in some other step of their gene expression. The repeat domains of Pufs appear to be sufficient for mRNA binding and decay regulation (Wharton et al. 1998; Jackson et al. 2004), yet these domains usually compose less than half of the protein. The large regions outside of the Puf repeat domain have no known function but may be acting in other cellular pathways. Together our work establishes the importance of direct testing using conventional approaches to evaluate candidate mRNA targets of Puf regulation derived from global microarray screens. This analysis not only identifies the bona fide targets of Pufmediated decay stimulation but also provides insight into the mechanisms by which Puf proteins act individually or in combination to regulate mRNA decay.

Several observations now argue that much of 3' UTRbased control of mRNAs will be combinatorial in nature. As we and others have shown, three out of five mRNA targets of yeast Puf regulation are controlled by multiple Puf proteins. Similarly, mRNAs in metazoan cells are regulated by multiple different miRNAs. The complicated nature of this combinatorial type of regulation implies that the effects of any given trans-acting factor may be minimized in an experiment since there are other contributing factors. These considerations may be complicating much of 3' UTR analysis.

\section{MATERIALS AND METHODS}

\section{Yeast strains}

The genotypes of the $S$. cerevisiae strains used are listed in Table 2.

The S. cerevisiae strains yWO102, yWO104, yWO105, yWO106, yWO198, yWO204, and yWO208 were obtained by mating. The parent haploid strains were crossed, and the diploids sporulated. The resulting tetrads were dissected, and each spore was genotyped. yWO48 was obtained by mating yWO7 with yWO14. yWO49 was obtained by mating yWO7 and yWO17. yWO102 was obtained by crossing yWO7 and yWO20. yWO15 and yWO49 were crossed to obtain yWO198. yWO3 and yWO7 were crossed 
TABLE 2. Strains used in this study

\begin{tabular}{|c|c|c|c|}
\hline Deletion & Strain & Genotype & Source \\
\hline Wild type & yWO3 & MATa, his4-539, leu2-3, lys2-201, trp1-1, ura3-52 & Hatfield et al. (1996); yRP683 \\
\hline Wild type & yWO7 & MAT $\alpha$, leu2-3, ura3-52, rpb1-1 & Caponigro et al. (1993); yRP693 \\
\hline puf $2 \Delta$ & yWO14 & $\begin{array}{l}\text { MATa, his4-539, leu2-3, trp1-1, ura3-52, } \\
\text { cup } 1:: \text { LEU2/PM, puf2::URA3 }\end{array}$ & Olivas and Parker (2000); yRP1237 \\
\hline puf $2 \Delta$ & yWO15 & $\begin{array}{l}\text { MATa, his4-539, leu2-3, trp1-1, ura3-52, } \\
\quad \text { cup } 1:: \text { LEU2/PM, puf }:: T R P 1\end{array}$ & This study \\
\hline puf5 & yWO17 & $\begin{array}{l}\text { MATa, his4-539, leu2-3, trp1-1, ura3-52, } \\
\text { cup } 1:: L E U 2 / P M, \text { puf5 }:: T R P 1\end{array}$ & Olivas and Parker (2000); yRP1240 \\
\hline puf1 & yWO20 & $\begin{array}{l}\text { MATa, his4-539, leu2-3, trp1-1, ura3-52, } \\
\text { cup } 1:: \text { LEU2/PM, puf1 ::NEO }\end{array}$ & Olivas and Parker (2000); yRP1243 \\
\hline puf4s & yWO22 & $\begin{array}{l}\text { MATa, leu2-3, lys2-201, trp1-1, ura3-52, } \\
\text { cup1::LEU2/PM, puf4::LYS2 }\end{array}$ & Olivas and Parker (2000); yRP1245 \\
\hline puf3s & yWO43 & $\begin{array}{l}\text { MAT } \alpha \text {, his4-539, leu2-3, trp1-1, ura3-52, rpb1-1, } \\
\quad \text { cup } 1:: \text { LEU2/PM, puf3 ::NEO }\end{array}$ & Olivas and Parker (2000); yRP1360 \\
\hline puf $2 \Delta$ & yWO48 & MAT $\alpha$, his4-539, leu2-3, ura3-52, rpb1-1, puf $:: U R A 3$ & This study \\
\hline puf5 & yWO49 & MAT $\alpha$, leu2-3, trp1-1, ura3-52, rpb1-1, puf5::URA3 & This study \\
\hline puf1s & yWO102 & MATa, leu2-3, trp1-1, ura3-52, cup $1::$ LEU2/PM, puf1 $:: N E O$ & This study \\
\hline Wild type & yWO104 & MATa, his4-539, leu2-3, lys2-201, ura3-52, rpb1-1 & This study \\
\hline puf4s & yWO105 & MAT $\alpha$, his4-539, lys2-201, ura3-52, rpb1-1, puf4::LYS2 & This study \\
\hline puf $4 \Delta$ & yWO106 & MATa, his4-539, leu2-3, lys2-201, rpb1-1, puf4::LYS2 & This study \\
\hline puf2 $2 \Delta$ puf5 & yWO198 & MAT $\alpha$, leu2-3, trp1-1, ura3-52, rpb1-1, puf2::TRP1, puf5::URA3 & This study \\
\hline$\Delta$ puf1-5 & yWO204 & $\begin{array}{l}\text { MAT } \alpha \text {, his4-539, leu2-3, lys2-201, trp1-1, ura3-52, rpb1-1, } \\
\text { puf1 ::NEO, puf } 2:: T R P 1, \text { puf3::NEO, puf4::LYS2, puf5::URA3 }\end{array}$ & This study \\
\hline puf $1 \Delta$ puf $5 \Delta$ & yWO208 & MATa, leu2-3, trp1-1, ura3-52, rpb1-1, puf1 ::NEO, puf5::URA3 & This study \\
\hline
\end{tabular}

to make yWO104, which was crossed to yWO22 to obtain yWO105 and yWO106. yWO208 and yWO204 were obtained by mating yWO102 and yWO49, and yWO28 and yWO106, respectively. The PUF deletions cause no obvious growth defects except the multiple PUF-deletion strains, which have subtle growth deficiencies.

\section{Pattern matching}

A program available through the Saccharomyces genome database, Yeast Genome Pattern Matching (http://seq.yeastgenome.org/ cgi-bin/SGD/PATMATCH/nph-patmatch), was used to identify mRNAs with potential Puf elements in their 3' UTRs. A search of UTR sequences containing UGUA followed by a 4-nt AU-rich region received nearly 30,000 hits.

\section{Site-directed mutagenesis}

Plasmids used in this study are listed in Table 3. Oligonucleotides used in this study are listed in Table 4 . In vitro site-directed mutagenesis was performed to mutate TIF1 3' UTR UGU regions using the QuikChange XL site-directed mutagenesis kit (Stratagene). To mutate TIF1 3' UTR UGUA site \#2, primers oWO310 and oWO311 were used in PCR-based mutagenesis of pWO53 and pWO70 as recommended by the manufacturer (Stratagene). To create MFA2/tif1-p3E 3' UTR in pWO109, site-directed mutagenesis was carried out with primers oWO430-431 in pWO88. All resulting mutants were confirmed by sequencing.

\section{In vivo decay analysis}

Decay of steady-state mRNA was monitored in strains containing the temperature-sensitive rpb1-1 RNA Polymerase II allele, in which transcription is rapidly repressed following a shift from $24^{\circ} \mathrm{C}$ to $37^{\circ} \mathrm{C}$. All yeast transformations were accomplished by LiOAc high-efficiency transformation (Gietz and Schiestl 1995).

Transcriptional shutoffs of the MFA2/TIF1 mRNA were performed in yeast strains containing pWO70 or pWO71. These plasmids express a fusion RNA containing the MFA2 coding region and TIF1 3' UTR with transcription regulated by the GAL UAS. pWO70 was made by PCR amplification of the TIF1 3' UTR from genomic DNA with primers oWO231 and oWO239. The PCR product was ligated into pWO24 between BglII and HindIII sites, replacing the $3^{\prime}$ UTR of MFA2 with that of TIF1. Similarly, the BglII/HindIII fragment was ligated into pWO54 (see below) to make pWO71.

Transcriptional shutoff assays of the MFA2/tif1 mRNA mutants were performed similarly to that of MFA2/TIF1. Creation of the MFA2/tif1-1 mutant (pWO53) occurred via a spontaneous error 84 nt from the stop codon in the PCR amplification of the TIF1 3' UTR and was confirmed by sequencing. Other MFA2/tif1 mutants were made by site-directed mutagenesis. To make pWO54, pWO61, pWO73, pWO89, and pWO110, the fragment containing the GAL-MFA2 3' UTR fusion was cut from pWO53, pWO72, pWO88, and pWO109 with PvuII and ligated into pWO58, which contains the LEU2 marker. pWO53, pWO70, pWO72, pWO88, and pWO109 (URA markers) were transformed into yWO7

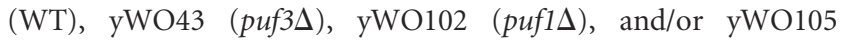
(puf4s) while pWO54, pWO71, pWO74, pWO89, and pWO110 were transformed into yWO48 (puf2s), yWO49 (puf5s), yWO205 ( $\Delta$ puf1-5), and/or yWO208 (puf1 $\Delta$ puf5 $\Delta$ ).

The HXK1 3' UTR was fused to PGK1 882 to create the PGK1/ HXK1 3' UTR fusion construct (pWO100). PGK1 82 is a truncated version of the stable PGK1 coding region that has been 
TABLE 3. Plasmids used in this study

\begin{tabular}{|c|c|c|c|}
\hline Plasmid & Description & Marker(s) & Source \\
\hline pWO21 & pBS-PUF2RD & $A M P$ & This study \\
\hline pWO22 & pGEX-PUF2RD & $A M P$ & This study \\
\hline pWO24 & pGAL-MFA2pG & URA3, AMP & Decker and Parker (1993); pRP485 \\
\hline pWO27 & pGAL-MFA2/HXK1 3' UTR & URA3, AMP & This study \\
\hline pWO48 & pBS-PUF1RD & $A M P$ & This study \\
\hline pWO49 & pGEX-PUF1RD & $A M P$ & This study \\
\hline pWO53 & pGAL-MFA2/tif1-1 $3^{\prime}$ UTR & URA3, AMP & This study \\
\hline pWO54 & pGAL-MFA2/tif1-1 $3^{\prime}$ UTR & $\angle E U 2, A M P$ & This study \\
\hline pWO58 & LEU, CEN Vector & $L E U 2, A M P$ & Brachmann et al. (1998); pRS415 \\
\hline pWO61 & pGAL-MFA2pG & $L E U 2, A M P$ & This study \\
\hline pWO70 & pGAL-MFA2/TIF1 3' UTR (WT) & URA3, AMP & This study \\
\hline pWO71 & pGAL-MFA2/TIF1 3' UTR (WT) & LEU2, AMP & This study \\
\hline pWO72 & pGAL-MFA2/tif1-2x 3' UTR & URA3, AMP & This study \\
\hline pWO73 & pGAL-MFA2/tif1-2x 3' UTR & $\angle E U 2, A M P$ & This study \\
\hline pWO88 & pGAL-MFA2/tif1-2 3' UTR & URA3, AMP & This study \\
\hline pWO89 & pGAL-MFA2/tif1-2 3' UTR & LEU2, AMP & This study \\
\hline pWO94 & pBS-HXK1 3' UTR & $A M P$ & This study \\
\hline pWO100 & pGAL-PGK1/HXK1 3' UTR & URA3, AMP & This study \\
\hline pWO101 & pGAL-PGK1/HXK1 3' UTR & LEU2, AMP & This study \\
\hline pWO102 & pGAL-PGK1 & URA3, AMP & Heaton et al. (1992); pRS227 \\
\hline pWO103 & pGAL-PGK1 & $L E U 2, A M P$ & This study \\
\hline pWO109 & pGAL-MFA2/tif1-p3E 3' UTR & URA3, AMP & This study \\
\hline pWO110 & pGAL-MFA2/tif1-p3E 3' UTR & $\angle E U 2, A M P$ & This study \\
\hline
\end{tabular}

shown to allow regulation of its mRNA decay rate by $3^{\prime}$ UTR regulatory sequences (Heaton et al. 1992). To create the PGK1/ HXK1 3' UTR construct, the HXK1 3' UTR was amplified from genomic DNA using primers oWO164 and oWO153. The BglII site at the $5^{\prime}$ end of the 530-nt product was first filled with Klenow (New England Biolabs). The product was then inserted between the Klenow-filled ClaI site and the HindIII site of pWO102 (PGK1 182 ) to create pWO100. The PGK1/HXK1 fragment was removed from pWO100 (SacI/HindIII) to pWO61, a LEU2 expression vector, to create pWO101. pWO100 and pWO101 express the PGK1 182 coding region fused to the HXK1 3' UTR under the control of the GAL UAS.

Control shutoff experiments of the native MFA2 or PGK1 mRNAs were performed using pWO24 and pWO61 (MFA2) or pWO102 and pWO103 (PGK1 882$)$. pWO61 was created by digesting pWO24 with PvuII and ligating the product containing

TABLE 4. Oligonucleotides used in this study

\begin{tabular}{|c|c|c|}
\hline Oligo & Description & Sequence \\
\hline oWO21 & $s c R I$ probe & gtctagccgcgaggaagg \\
\hline oWO105 & HXK1 probe & cataagggcatcactcataag \\
\hline oWO136 & PUF2RD up primer & cgcggatcccctccaccatcattatcggatagt \\
\hline oWO137 & PUF2RD down primer & tctgcccgggaaacagaaacgcctctggc \\
\hline oWO144 & PUF1RD up primer & cccggatccgaattcgcaaattccgatgaataccaaatcaattcg \\
\hline oWO145 & PUF1RD down primer & cccccgccggcgcagctgcgaaatgctgctgttatgatgctgc \\
\hline oWO153 & HXK1 3' UTR down primer & ccgaagcttccgagctatcctacgactttc \\
\hline oWO164 & HXK1 3' UTR up primer & gccagatctcttggtatcattggcgcttaatg \\
\hline oWO231 & TIF1 3' UTR down primer & ccgaagcttctctatacaaggcagaggg \\
\hline oWO238 & MFA2 probe & atattgattagatcaggaattcc \\
\hline oWO239 & TIF1 3' UTR up primer & ccgaagcttctctatacaaggcagaggg \\
\hline oWO249 & TIF1 3' UTR probe & caaccttcgtgccgagagtc \\
\hline oWO310 & TIF1 SDM primer \#1 & ggttgaaataccctatactaattgtttgctttctcttttacactatatccgaacgtatctatctgaaattttc \\
\hline oWO311 & TIF1 SDM primer \#2 & gaaaaatttcagatagatacgttcggatatagtgtaaaagacaaagcaaacaattagtatagggtatttcaacc \\
\hline oWO430 & TIF1 SDM P3E up primer & ctaaaaagttatatatgcttcttgtatatatattgttttctttttacattcctattattcttcaaaagtccaaaagactc \\
\hline oWO431 & TIF1 SDM P3E down primer & gagtctttggactttgaagaataataggaatgtaaaaagaaaaacaatatatatacaagaagcatatataactttttag \\
\hline
\end{tabular}


GAL-MFA2 into pWO58. pWO103 was created by inserting the PGK1 fragment from pWO102 into SacI/HindIII sites of pWO61. pWO61 and pWO103 were transformed into yWO48, yWO49, yWO205, and yWO208, while pWO24 and pWO103 were transformed into yWO7, yWO102, and yWO105, yWO205 and yWO208. Decay of endogenously transcribed HXK1 mRNA was detected by stripping the MFA2 control or MFA2/tif1 Northern blots and re-probing for $H X K 1$ mRNA.

Transcriptional shutoff experiments were performed essentially as described (Caponigro et al. 1993) with the following modifications: $200 \mathrm{~mL}$ cultures were grown to an $\mathrm{OD}_{600}$ of 1.0 in synthetic media with $2 \%$ galactose. Half of each culture was harvested and resuspended in $20 \mathrm{~mL}$ of $37^{\circ} \mathrm{C}$ media containing $8 \%$ dextrose shutting off transcription via both the temperature-sensitive inactivation of RNA-pol II and the carbon source inactivation of the GAL promoter. Northern blots were probed with the following ${ }^{32} \mathrm{P}$-end-labeled oligonucleotides complementary to 3 ' UTR sequences: oWO238 (MFA2), oWO249 (TIF1), and oWO105 (HXK1). Total RNA was isolated from yeast as described (Caponigro et al. 1993), and Northern blots were prepared (NytranSupercharge membrane, Schleicher and Schuell). All blots were normalized for loading to $s c R I$ RNA, a constitutively expressed RNA Polymerase III transcript (Felici et al. 1989). All quantification of RNA was accomplished using ImageQuant software (Molecular Dynamics).

\section{Protein purification}

The GST-PUF3RD and GST-PUF5RD constructs in pGEX-6P-1 (Amersham Biosciences) were previously created (Jackson et al. 2004). The GST-PUF1RD fusion construct was created by PCRamplification of an 1140-nt region of genomic PUF1 (amino acids 551-934) using the primers oWO144 and oWO145. The PCR product was inserted into pBlueScript (Stratagene) between BamHI and Not1 to yield pWO48. pWO48 was digested with BamHI and PvuII then cloned into pGEX-6P-3 (Amersham Biosciences) between BamHI and SmaI to create pWO49, the GST-Puf1RDp expression vector. To create the GST-PUF2RD fusion construct, nucleotides 1453-2712 were amplified from genomic PUF2 (encoding amino acids 485-904) with primers oWO136 and oWO137. This product was inserted into pBlueScript between the BamHI and XmaI sites, creating pWO21. The BamHI-XmaI digestion product of pWO21 was then ligated into pGEX-6P-3 to yield the GST-Puf2RDp expression vector pWO22. Each construct was verified by sequencing. The GST fusion constructs were transformed into BL-21 protease-deficient E. coli and purified as recommended (Amersham Biosciences). Eluates were dialyzed in $50 \mathrm{mM}$ Tris- $\mathrm{HCl}, \mathrm{pH} 8.0$, and verified by Western analysis with anti-GST antibodies.

\section{In vitro binding assays}

In vitro transcribed RNA containing the $3^{\prime}$ UTR of $H X K 1$ mRNA was made by first amplifying the HXK1 3' UTR with primers oWO153 and oWO164, then ligating the fragment into pBlueScript between BamHI and HindIII sites. The plasmid (pWO94) was digested with HpaII or SspI prior to transcription. RNA was transcribed using T3 RNA polymerase (Ambion) in the presence of ${ }^{32} \mathrm{P}$ UTP to produce transcripts $117 \mathrm{nt}$ and $58 \mathrm{nt}$ in length. The resulting transcripts were treated with DNaseI (Promega) and then purified by separation on denaturing polyacrylamide gel, elution from gel slice, and ethanol precipitation. Binding was performed essentially as described (Olivas and Parker 2000) with radiolabeled transcript $(100,000 \mathrm{cpm})$ in the presence or absence of GST-Puf1RDp $(2 \mu \mathrm{M})$, GST-Puf2RDp $(2 \mu \mathrm{M})$, GST-Puf3RDp (3 $\mu \mathrm{M})$, or GST-Puf5RDp $(2 \mu \mathrm{M})$.

\section{Hxk1p enzyme assay}

Yeast strains yWO7 (wild-type), yWO48 (puf2s), yWO49

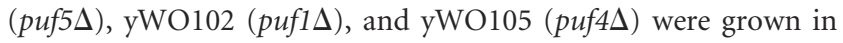
synthetic media with $2 \%$ dextrose to $\mathrm{OD}_{600}$ of 1.0 , harvested, and washed twice with media alone. Extracts were prepared as described (Kawasaki and Fraenkel 1982). Total protein was determined (Bio-Rad Protein Assay, Bio-Rad). Detection of fructose phosphorylation by hexokinase was monitored as described (Walsh et al. 1991) by adding extract containing 50 $\mu \mathrm{g}$ of total protein to reaction buffer ( $5 \mathrm{mM}$ triethanolamine, 10 $\mathrm{mM} \mathrm{MgCl}_{2}$ ( $\mathrm{pH}$ 7.4), $0.3 \mathrm{mM}$ NADP, $1 \mathrm{mM} \mathrm{rATP,} 5 \mathrm{mM}$ fructose) with $2 \mu \mathrm{g}$ of phosphoglucose isomerase (Roche) and 4 $\mu \mathrm{g}$ of glucose-6-P dehydrogenase (Roche). Enzyme activity (U/ $\mathrm{mg}$ ) was calculated according to the change in absorbance at 340 $\mathrm{nm}$ with extract alone as the standard.

\section{ACKNOWLEDGMENTS}

We gratefully thank Bethany Zolman, Florencia Lopez Leban, and Melanie Miller for helpful discussions and review of the manuscript. This work was supported by a grant to W.M.O from the National Institutes of Health (GM63759).

Received September 26, 2007; accepted November 6, 2007.

\section{REFERENCES}

Adams, C.D. and Gross, D.S. 1991. The yeast heat shock response is induced by conversion of cells to spheroplasts and by potent transcriptional inhibitors. J. Bacteriol. 173: 7429-7435.

Aragon, A.D., Quinones, G.A., Thomas, E.V., Roy, S., and WernerWashburne, M. 2006. Release of extraction-resistant mRNA in stationary phase Saccharomyces cerevisiae produces a massive increase in transcript abundance in response to stress. Genome Biol. 7: R9. doi: 10.1186/gb-2006-7-2-r9.

Bachorik, J.L. and Kimble, J. 2005. Redundant control of the Caenorhabditis elegans sperm/oocyte switch by PUF-8 and FBF1, two distinct Puf RNA-binding proteins. Proc. Natl. Acad. Sci. 102: 10893-10897.

Bernstein, D., Hook, B., Hajarnavis, A., Opperman, L., and Wickens, M. 2005. Binding specificity and mRNA targets of a $C$. elegans PUF protein, FBF-1. RNA 11: 447-458.

Boy-Marcotte, E., Perrot, M., Bussereau, F., Boucherie, H., and Jacquet, M. 1998. Msn2p and Msn4p control a large number of genes induced at the diauxic transition which are repressed by cyclic AMP in Saccharomyces cerevisiae. J. Bacteriol. 180: 10441052.

Brachmann, C.B., Davies, A., Cost, G.J., Caputo, E., Li, J., Hieter, P., and Boeke, J.D. 1998. Designer deletion strains derived from Saccharomyces cerevisiae S288C: A useful set of strains and plasmids for PCR-mediated gene disruption and other applications. Yeast 14: 115-132.

Caponigro, G., Muhlrad, D., and Parker, R. 1993. A small segment of the MATal transcript promotes mRNA decay in Saccharomyces 
cerevisiae: A stimulatory role for rare codons. Mol. Cell. Biol. 13: 5141-5148.

Crittenden, S.L., Bernstein, D.S., Bachorik, J.L., Thompson, B.E., Gallegos, M., Petcherski, A.G., Moulder, G., Barstead, R., Wickens, M., and Kimble, J. 2002. A conserved RNA-binding protein controls germline stem cells in Caenorhabditis elegans. Nature 417: 660-663.

Curtis, D., Treiber, D.K., Tao, F., Zamore, P.D., Williamson, J.R., and Lehmann, R. 1997. A CCHC metal-binding domain in Nanos is essential for translation regulation. EMBO J. 16: 834-843.

Decker, C.J. and Parker, R. 1993. A turnover pathway for both stable and unstable mRNAs in yeast: Evidence for a requirement for deadenylation. Genes \& Dev. 7: 1632-1643.

Derrigo, M., Cestelli, A., Savettieri, G., and Di Liegro, I. 2000. RNAprotein interactions in the control of stability and localization of messenger RNA. Int. J. Mol. Med. 5: 111-123.

Edwards, T.A., Pyle, S.E., Wharton, R.P., and Aggarwal, A.K. 2001. Structure of Pumilio reveals similarity between RNA and peptide binding motifs. Cell 105: 281-289.

Fehrenbacher, K.L., Boldogh, I.R., and Pon, L.A. 2005. A role for Jsnlp in recruiting the Arp2/3 complex to mitochondria in budding yeast. Mol. Biol. Cell 16: 5094-5102.

Felici, F., Cesareni, G., and Hughes, J.M.X. 1989. The most abundant small cytoplasmic RNA of Saccharomyces cerevisiae has an important function required for normal cell growth. Mol. Cell. Biol. 9: 3260-3268.

Foat, B.C., Houshmandi, S.S., Olivas, W.M., and Bussemaker, H.J. 2005. Profiling condition-specific, genome-wide regulation of mRNA stability in yeast. Proc. Natl. Acad. Sci. 102: 17675-17680.

Forbes, A. and Lehmann, R. 1998. Nanos and Pumilio have critical roles in the development and function of Drosophila germline stem cells. Development 125: 679-690.

Gamberi, C., Peterson, D.S., He, L., and Gottlieb, E. 2002. An anterior function for the Drosophila posterior determinant Pumilio. Development 129: 2699-2710.

Gancedo, J.M., Clifton, D., and Fraenkel, D.G. 1977. Yeast hexokinase mutants. J. Biol. Chem. 252: 4443-4444.

Garcia-Rodriguez, L.J., Gay, A.C., and Pon, L.A. 2007. Puf3p, a Pumilio family RNA binding protein, localizes to mitochondria and regulates mitochondrial biogenesis and motility in budding yeast. J. Cell Biol. 176: 197-207.

Gerber, A.P., Herschlag, D., and Brown, P.O. 2004. Extensive association of functionally and cytotopically related mRNAs with Puf family RNA-binding proteins in yeast. PLoS Biol. 2: 342-354. doi: 10.1371/journal.pbio.0020079.

Gietz, R.D. and Schiestl, R.H. 1995. Transforming yeast with DNA. Methods Mol. Cell. Biol. 5: 255-269.

Goldstrohm, A.C., Hook, B.A., Seay, D.J., and Wickens, M. 2006. PUF proteins bind Pop2p to regulate messenger RNAs. Nat. Struct. Mol. Biol. 13: 533-539.

Grigull, J., Mnaimneh, S., Pootoolal, J., Robinson, M.D., and Hughes, T.R. 2004. Genome-wide analysis of mRNA stability using transcription inhibitors and microarrays reveals posttranscriptional control of ribosome biogenesis factors. Mol. Cell. Biol. 24: 5534-5547.

Grzybowska, E.A., Wilczynska, A., and Siedlecki, J.A. 2001. Regulatory functions of 3' UTRs. Biochem. Biophys. Res. Commun. 288: 291-295.

Gu, W., Deng, Y., Zenklusen, D., and Singer, R.H. 2004. A new yeast PUF family protein, Puf6p, represses ASH1 mRNA translation and is required for its localization. Genes \& Dev. 18: 1452-1465.

Guhaniyogi, J. and Brewer, G. 2001. Regulation of mRNA stability in mammalian cells. Gene 265: 11-23.

Hatfield, L., Beelman, C.A., Stevens, A., and Parker, R. 1996. Mutations in trans-acting factors affecting mRNA decapping in Saccharomyces cerevisiae. Mol. Cell. Biol. 16: 5830-5838.

Heaton, B., Decker, C., Muhlrad, D., Donahue, J., Jacobson, A., and Parker, R. 1992. Analysis of chimeric mRNAs derived from the STE3 mRNA identifies multiple regions within yeast mRNAs that modulate mRNA decay. Nucleic Acids Res. 20: 5365-5373. doi: $10.1093 /$ nar/20.20.5365.

Herrick, D., Parker, R., and Jacobson, A. 1990. Identification and comparison of stable and unstable mRNAs in Saccharomyces cerevisiae. Mol. Cell. Biol. 10: 2269-2284.

Hook, B.A., Goldstrohm, A.C., Saeu, D.J., and Wickens, M. 2007. Two yeast Puf proteins negatively regulate a single mRNA. J. Biol. Chem. 282: $15430-15438$.

Houshmandi, S.S. and Olivas, W.M. 2005. Yeast Puf3 mutants reveal the complexity of Puf-RNA binding and identify a loop required for regulation of mRNA decay. RNA 11: 1655-1666.

Jackson, J.S., Houshmandi, S.S., Leban, F.L., and Olivas, W.M. 2004. Recruitment of the Puf3 protein to its mRNA target for regulation of mRNA decay in yeast. RNA 10: 1625-1636.

Kawasaki, G. and Fraenkel, D.G. 1982. Cloning of yeast glycolysis genes by complementation. Biochem. Biophys. Res. Commun. 108: 1107-1112.

Kraemer, B., Crittenden, S., Gallegos, M., Moulder, G., Barstead, R., Kimble, J., and Wickens, M. 1999. NANOS-3 and FBF proteins physically interact to control the sperm-oocyte switch in Caenorhabditis elegans. Curr. Biol. 9: 1009-1018.

Lidder, P., Gutierrez, R.A., Salome, P.A., McClung, C.R., and Green, P.J. 2005. Circadian control of messenger RNA stability. Association with a sequence-specific messenger RNA decay pathway. Plant Physiol. 138: 2374-2385.

Menon, K.P., Sanyal, S., Habara, Y., Sanchez, R., Wharton, R.P., Ramaswami, M., and Zinn, K. 2004. The translational repressor Pumilio regulates presynaptic morphology and controls postsynaptic accumulation of translation factor eIF-4E. Neuron 44: 663-676.

Moore, F.L., Jaruzelska, J., Fox, M.S., Urano, J., Firpo, M.T., Turek, P.J., Dorfman, D.M., and Pera, R.A. 2003. Human Pumilio-2 is expressed in embryonic stem cells and germ cells and interacts with DAZ (deleted in AZoospermia) and DAZ-like proteins. Proc. Natl. Acad. Sci. 100: 538-543.

Murata, Y. and Wharton, R. 1995. Binding of Pumilio to maternal hunchback mRNA is required for posterior patterning in Drosophila embryos. Cell 80: 747-756.

Nakahata, S., Kotani, T., Mita, K., Kawasaki, T., Katsu, Y., Nagahama, Y., and Yamashita, M. 2003. Involvement of Xenopus Pumilio in the translational regulation that is specific to cyclin B1 mRNA during oocyte maturation. Mech. Dev. 120: 865-880.

Olivas, W. and Parker, R. 2000. The Puf3 protein is a transcript-specific regulator of mRNA degradation in yeast. EMBO J. 19: 6602-6611.

Parisi, M. and Lin, H. 1999. The Drosophila Pumilio gene encodes two functional protein isoforms that play multiple roles in germline development, gonadogenesis, oogenesis, and embryogenesis. Genetics 153: 235-250.

Parker, R. and Song, H. 2004. The enzymes and control of eukaryotic mRNA turnover. Nat. Struct. Mol. Biol. 11: 121-127.

Penelova, A., Richman, L., Neupert, B., Simanis, V., and Kuhn, L.C. 2005. Analysis of the contribution of changes in mRNA stability to the changes in steady-state levels of cyclin mRNA in the mammalian cell cycle. FEBS J. 272: 5217-5229.

Rodriguez, A., De La Cera, T., Herrero, P., and Moreno, F. 2001. The hexokinase 2 protein regulates the expression of the GLK1, HXK1, and HXK2 genes of Saccharomyces cerevisiae. Biochem. J. 355: 625-631.

Shim, J. and Karin, M. 2002. The control of mRNA stability in response to extracellular stimuli. Mol. Cells 14: 323-331.

Sonoda, J. and Wharton, R.P. 1999. Recruitment of Nanos to hunchback mRNA by Pumilio. Genes \& Dev. 13: 2704-2712.

Sonoda, J. and Wharton, R.P. 2001. Drosophila brain tumor is a translational repressor. Genes \& Dev. 15: 762-773.

Tadauchi, T., Matsumoto, K., Herskowitz, I., and Irie, K. 2001. Posttranscriptional regulation through the HO 3'-UTR by Mpt5, a yeast homolog of Pumilio and FBF. EMBO J. 20: 552-561.

Taylor, R., Kebaara, B.W., Nazarenus, T., Jones, A., Yamanaka, R., Uhrenholdt, R., Wendler, J.P., and Atkin, A.L. 2005. Gene set 
coregulated by the Saccharomyces cerevisiae nonsense-mediated mRNA decay pathway. Eukaryot. Cell 4: 2066-2077.

Walsh, R.B., Clifton, D., Horak, J., and Fraenkel, D.G. 1991. Saccharomyces cerevisiae null mutants in glucose phosphorylation: Metabolism and invertase expression. Genetics 128: 521-527.

Wang, X., McLachlan, J., Zamore, P.D., and Tanaka Hall, T.M. 2002. Modular recognition of RNA by a human Pumilio-homology domain. Cell 110: 501-512.

Wharton, R.P. and Struhl, G. 1991. RNA regulatory elements mediate control of Drosophila body pattern by the posterior morphogen nanos. Cell 67: 955-967.

Wharton, R.P., Sonoda, J., Lee, T., Patterson, M., and Murata, Y. 1998. The Pumilio RNA-binding domain is also a translational regulator. Mol. Cell 1: 863-872.
Wickens, M., Bernstein, D.S., Kimble, J., and Parker, R. 2002. A PUF family portrait: 3' UTR regulation as a way of life. Trends Genet. 18: $150-157$.

Wreden, C., Verrotti, A.C., Schisa, J.A., Lieberfarb, M.E., and Strickland, S. 1997. Nanos and pumilio establish embryonic polarity in Drosophila by promoting posterior deadenylation of hunchback mRNA. Development 124: 3015-3023.

Ye, B., Petritsch, C., Clark, I.E., Gavis, E.R., Jan, L.Y., and Jan, Y.N. 2004. Nanos and Pumilio are essential for dendrite morphogenesis in Drosophila peripheral neurons. Curr. Biol. 14: 314-321.

Zhang, B., Gallegos, M., Puoti, A., Durkin, E., Fields, S., Kimble, J., and Wickens, M.P. 1997. A conserved RNA-binding protein that regulates sexual fates in the $C$. elegans hermaphrodite germ line. Nature 390: 477-484. 

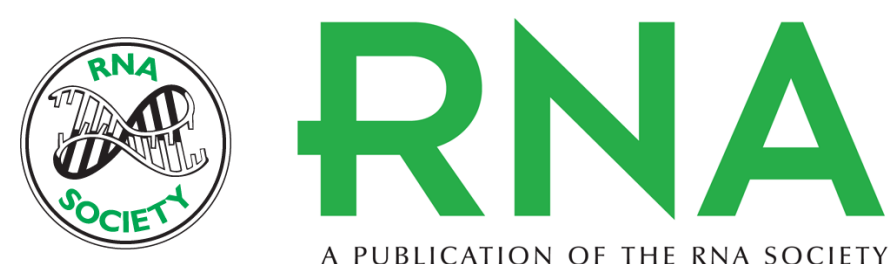

A PUBLICATION OF THE RNA SOCIETY

\section{Puf1p acts in combination with other yeast Puf proteins to control mRNA stability}

Randi J. Ulbricht and Wendy M. Olivas

RNA 2008 14: 246-262

References This article cites 57 articles, 31 of which can be accessed free at:

http://rnajournal.cshlp.org/content/14/2/246.full.html\#ref-list-1

\section{License}

Email Alerting Receive free email alerts when new articles cite this article - sign up in the box at the Service top right corner of the article or click here. 